\title{
Theoretical Determination of Rate Constants from Excited-State: Application to Benzophenone
}

Katsuyuki Shizu and Hironori Kaji*

Institute for Chemical Research, Kyoto University, Uji, Kyoto 611-0011, Japan 
Table S1. Standard nuclear orientation of the optimized $\mathrm{S}_{0}$ geometry of BP optimized at the B3LYP/6-31G(d) level of theory.

\begin{tabular}{llllr}
\hline \hline Atom & Element symbol & $\mathrm{x}(\AA)$ & $\mathrm{y}(\AA)$ & $\mathrm{z}(\AA)$ \\
\hline 1 & $\mathrm{C}$ & 0.000000 & 0.000000 & 1.079729 \\
2 & $\mathrm{O}$ & 0.000000 & 0.000000 & 2.306126 \\
3 & $\mathrm{C}$ & 0.000000 & 1.304256 & 0.336843 \\
4 & $\mathrm{C}$ & 0.000000 & -1.304256 & 0.336843 \\
5 & $\mathrm{C}$ & -0.589406 & 1.466202 & -0.926507 \\
6 & $\mathrm{C}$ & -0.618323 & 2.721851 & -1.534707 \\
7 & $\mathrm{C}$ & -0.048485 & 3.823125 & -0.894323 \\
8 & $\mathrm{C}$ & 0.534849 & 3.671797 & 0.367554 \\
9 & $\mathrm{C}$ & 0.546670 & 2.424378 & 0.983822 \\
10 & $\mathrm{C}$ & 0.589406 & -1.466202 & -0.926507 \\
11 & $\mathrm{C}$ & 0.618323 & -2.721851 & -1.534707 \\
12 & $\mathrm{C}$ & 0.048485 & -3.823125 & -0.894323 \\
13 & $\mathrm{C}$ & -0.534849 & -3.671797 & 0.367554 \\
14 & $\mathrm{C}$ & -0.546670 & -2.424378 & 0.983822 \\
15 & $\mathrm{H}$ & -1.049155 & 0.617643 & -1.422898 \\
16 & $\mathrm{H}$ & -1.088841 & 2.839470 & -2.507095 \\
17 & $\mathrm{H}$ & -0.064087 & 4.798694 & -1.373103 \\
18 & $\mathrm{H}$ & 0.973234 & 4.529273 & 0.870906 \\
19 & $\mathrm{H}$ & 0.974394 & 2.292099 & 1.972403 \\
20 & $\mathrm{H}$ & 1.049155 & -0.617643 & -1.422898 \\
21 & $\mathrm{H}$ & 1.088841 & -2.839470 & -2.507095 \\
22 & $\mathrm{H}$ & 0.064087 & -4.798694 & -1.373103 \\
23 & $\mathrm{H}$ & -0.973234 & -4.529273 & 0.870906 \\
24 & $\mathrm{H}$ & -0.974394 & -2.292099 & 1.972403 \\
\hline \hline & & & & \\
\hline
\end{tabular}


Table S2. Standard nuclear orientation of the optimized $\mathrm{S}_{1}$ geometry of BP optimized at the TDB3LYP/6-31G(d) level of theory.

\begin{tabular}{llllr}
\hline \hline Atom & Element symbol & $\mathrm{x}(\AA)$ & $\mathrm{y}(\AA)$ & $\mathrm{z}(\AA)$ \\
\hline 1 & $\mathrm{C}$ & 0.000000 & 0.000000 & 0.938540 \\
2 & $\mathrm{O}$ & 0.000000 & 0.000000 & 2.260490 \\
3 & $\mathrm{C}$ & 0.000000 & 1.300410 & 0.306348 \\
4 & $\mathrm{C}$ & 0.000000 & -1.300410 & 0.306348 \\
5 & $\mathrm{C}$ & -0.474841 & 1.506284 & -1.014347 \\
6 & $\mathrm{C}$ & -0.470460 & 2.777620 & -1.571285 \\
7 & $\mathrm{C}$ & -0.021240 & 3.882473 & -0.836035 \\
8 & $\mathrm{C}$ & 0.419765 & 3.698282 & 0.478053 \\
9 & $\mathrm{C}$ & 0.432392 & 2.431849 & 1.048255 \\
10 & $\mathrm{C}$ & 0.474841 & -1.506284 & -1.014347 \\
11 & $\mathrm{C}$ & 0.470460 & -2.777620 & -1.571285 \\
12 & $\mathrm{C}$ & 0.021240 & -3.882473 & -0.836035 \\
13 & $\mathrm{C}$ & -0.419765 & -3.698282 & 0.478053 \\
14 & $\mathrm{C}$ & -0.432392 & -2.431849 & 1.048255 \\
15 & $\mathrm{H}$ & -0.881267 & 0.674658 & -1.578166 \\
16 & $\mathrm{H}$ & -0.839732 & 2.913807 & -2.584444 \\
17 & $\mathrm{H}$ & -0.024323 & 4.873485 & -1.280358 \\
18 & $\mathrm{H}$ & 0.763794 & 4.549104 & 1.060293 \\
19 & $\mathrm{H}$ & 0.801856 & 2.295433 & 2.059161 \\
20 & $\mathrm{H}$ & 0.881267 & -0.674658 & -1.578166 \\
21 & $\mathrm{H}$ & 0.839732 & -2.913807 & -2.584444 \\
22 & $\mathrm{H}$ & 0.024323 & -4.873485 & -1.280358 \\
23 & $\mathrm{H}$ & -0.763794 & -4.549104 & 1.060293 \\
24 & $\mathrm{H}$ & -0.801856 & -2.295433 & 2.059161 \\
\hline \hline & & & & \\
\hline
\end{tabular}


Table S3. Standard nuclear orientation of the optimized $\mathrm{T}_{1}$ geometry of BP optimized at the TDB3LYP/6-31G(d) level of theory.

\begin{tabular}{llllr}
\hline \hline Atom & Element symbol & $\mathrm{x}(\AA)$ & $\mathrm{y}(\AA)$ & $\mathrm{z}(\AA)$ \\
\hline 1 & $\mathrm{C}$ & 0.000000 & 0.000000 & 0.928471 \\
2 & $\mathrm{O}$ & 0.000000 & 0.000000 & 2.260868 \\
3 & $\mathrm{C}$ & 0.000000 & 1.299716 & 0.307992 \\
4 & $\mathrm{C}$ & 0.000000 & -1.299716 & 0.307992 \\
5 & $\mathrm{C}$ & -0.536717 & 1.516040 & -0.990967 \\
6 & $\mathrm{C}$ & -0.534561 & 2.783387 & -1.552187 \\
7 & $\mathrm{C}$ & -0.037304 & 3.884819 & -0.837068 \\
8 & $\mathrm{C}$ & 0.459455 & 3.694856 & 0.457943 \\
9 & $\mathrm{C}$ & 0.478490 & 2.430625 & 1.030161 \\
10 & $\mathrm{C}$ & 0.536717 & -1.516040 & -0.990967 \\
11 & $\mathrm{C}$ & 0.534561 & -2.783387 & -1.552187 \\
12 & $\mathrm{C}$ & 0.037304 & -3.884819 & -0.837068 \\
13 & $\mathrm{C}$ & -0.459455 & -3.694856 & 0.457943 \\
14 & $\mathrm{C}$ & -0.478490 & -2.430625 & 1.030161 \\
15 & $\mathrm{H}$ & -0.968950 & 0.683108 & -1.535491 \\
16 & $\mathrm{H}$ & -0.940266 & 2.925214 & -2.550552 \\
17 & $\mathrm{H}$ & -0.046369 & 4.875322 & -1.282362 \\
18 & $\mathrm{H}$ & 0.844746 & 4.541324 & 1.020453 \\
19 & $\mathrm{H}$ & 0.887005 & 2.284238 & 2.023820 \\
20 & $\mathrm{H}$ & 0.968950 & -0.683108 & -1.535491 \\
21 & $\mathrm{H}$ & 0.940266 & -2.925214 & -2.550552 \\
22 & $\mathrm{H}$ & 0.046369 & -4.875322 & -1.282362 \\
23 & $\mathrm{H}$ & -0.844746 & -4.541324 & 1.020453 \\
24 & $\mathrm{H}$ & -0.887005 & -2.284238 & 2.023820 \\
\hline \hline & & & & \\
\hline
\end{tabular}


Table S4. Standard nuclear orientation of the optimized $\mathrm{T}_{3}$ geometry of BP optimized at the TDB3LYP/6-31G(d) level of theory.

\begin{tabular}{llllr}
\hline \hline Atom & Element symbol & $\mathrm{x}(\AA)$ & $\mathrm{y}(\AA)$ & $\mathrm{z}(\AA)$ \\
\hline 1 & $\mathrm{C}$ & 0.000000 & 0.000000 & 1.022513 \\
2 & $\mathrm{O}$ & 0.000000 & 0.000000 & 2.269411 \\
3 & $\mathrm{C}$ & 0.000000 & 1.292412 & 0.301994 \\
4 & $\mathrm{C}$ & 0.000000 & -1.292412 & 0.301994 \\
5 & $\mathrm{C}$ & -0.472718 & 1.495703 & -1.037384 \\
6 & $\mathrm{C}$ & -0.498384 & 2.759048 & -1.592517 \\
7 & $\mathrm{C}$ & -0.051993 & 3.892096 & -0.848748 \\
8 & $\mathrm{C}$ & 0.384798 & 3.714753 & 0.487586 \\
9 & $\mathrm{C}$ & 0.396876 & 2.459425 & 1.058094 \\
10 & $\mathrm{C}$ & 0.472718 & -1.495703 & -1.037384 \\
11 & $\mathrm{C}$ & 0.498384 & -2.759048 & -1.592517 \\
12 & $\mathrm{C}$ & 0.051993 & -3.892096 & -0.848748 \\
13 & $\mathrm{C}$ & -0.384798 & -3.714753 & 0.487586 \\
14 & $\mathrm{C}$ & -0.396876 & -2.459425 & 1.058094 \\
15 & $\mathrm{H}$ & -0.854879 & 0.656084 & -1.606372 \\
16 & $\mathrm{H}$ & -0.871075 & 2.896974 & -2.603844 \\
17 & $\mathrm{H}$ & -0.059867 & 4.878822 & -1.300907 \\
18 & $\mathrm{H}$ & 0.706271 & 4.576595 & 1.066149 \\
19 & $\mathrm{H}$ & 0.703141 & 2.301830 & 2.085648 \\
20 & $\mathrm{H}$ & 0.854879 & -0.656084 & -1.606372 \\
21 & $\mathrm{H}$ & 0.871075 & -2.896974 & -2.603844 \\
22 & $\mathrm{H}$ & 0.059867 & -4.878822 & -1.300907 \\
23 & $\mathrm{H}$ & -0.706271 & -4.576595 & 1.066149 \\
24 & $\mathrm{H}$ & -0.703141 & -2.301830 & 2.085648 \\
\hline \hline & & & & \\
\hline
\end{tabular}


Table S5. Standard nuclear orientation of the optimized $\mathrm{T}_{1}$ geometry of BP optimized at the UB3LYP/6-31G(d) level of theory.

\begin{tabular}{llllr}
\hline \hline Atom & Element symbol & $\mathrm{x}(\AA)$ & $\mathrm{y}(\AA)$ & $\mathrm{z}(\AA)$ \\
\hline 1 & $\mathrm{C}$ & 0.000000 & 0.000000 & 0.920083 \\
2 & $\mathrm{O}$ & 0.000000 & 0.000000 & 2.249568 \\
3 & $\mathrm{C}$ & 0.000000 & 1.306871 & 0.300164 \\
4 & $\mathrm{C}$ & 0.000000 & -1.306871 & 0.300164 \\
5 & $\mathrm{C}$ & -0.536493 & 1.526166 & -0.994373 \\
6 & $\mathrm{C}$ & -0.536945 & 2.798290 & -1.548315 \\
7 & $\mathrm{C}$ & -0.043661 & 3.895201 & -0.826394 \\
8 & $\mathrm{C}$ & 0.455362 & 3.699000 & 0.465386 \\
9 & $\mathrm{C}$ & 0.479611 & 2.429199 & 1.027923 \\
10 & $\mathrm{C}$ & 0.536493 & -1.526166 & -0.994373 \\
11 & $\mathrm{C}$ & 0.536945 & -2.798290 & -1.548315 \\
12 & $\mathrm{C}$ & 0.043661 & -3.895201 & -0.826394 \\
13 & $\mathrm{C}$ & -0.455362 & -3.699000 & 0.465386 \\
14 & $\mathrm{C}$ & -0.479611 & -2.429199 & 1.027923 \\
15 & $\mathrm{H}$ & -0.963427 & 0.694659 & -1.545203 \\
16 & $\mathrm{H}$ & -0.940716 & 2.944637 & -2.546849 \\
17 & $\mathrm{H}$ & -0.055331 & 4.888506 & -1.265587 \\
18 & $\mathrm{H}$ & 0.838677 & 4.542592 & 1.033510 \\
19 & $\mathrm{H}$ & 0.892619 & 2.275350 & 2.019266 \\
20 & $\mathrm{H}$ & 0.963427 & -0.694659 & -1.545203 \\
21 & $\mathrm{H}$ & 0.940716 & -2.944637 & -2.546849 \\
22 & $\mathrm{H}$ & 0.055331 & -4.888506 & -1.265587 \\
23 & $\mathrm{H}$ & -0.838677 & -4.542592 & 1.033510 \\
24 & $\mathrm{H}$ & -0.892619 & -2.275350 & 2.019266 \\
\hline \hline & & & & \\
\hline
\end{tabular}




\section{B3LYP/6-31G(d)}

\section{$S_{0}$ geometry}

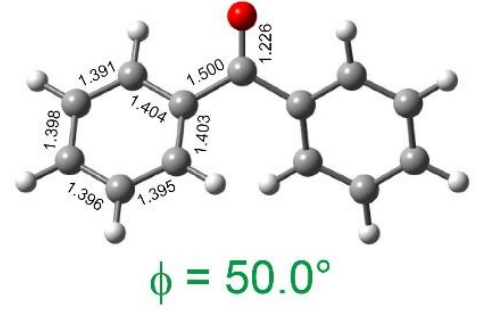

TD-B3LYP/6-31G(d)

\section{$\mathrm{S}_{1}$ geometry}

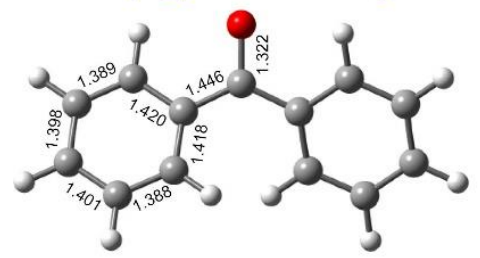

$\phi=39.6^{\circ}$

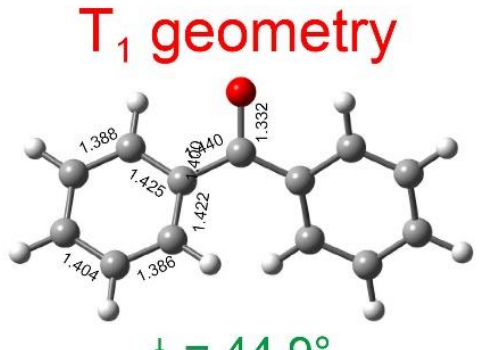

$\phi=44.9^{\circ}$

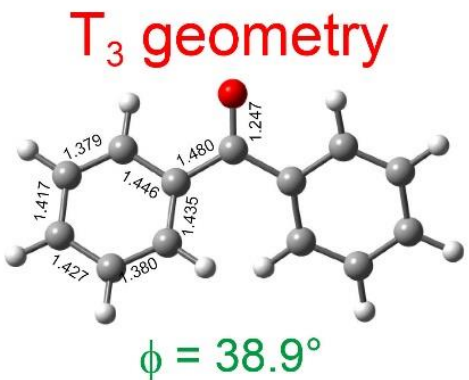

\section{UB3LYP/6-31G(d)}

$\mathrm{T}_{3}$ geometry

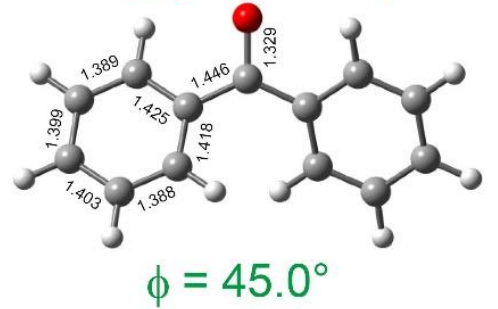

Figure S1. The optimized $\mathrm{S}_{0}, \mathrm{~S}_{1}, \mathrm{~T}_{1}$, and $\mathrm{T}_{3}$ geometries. The $\mathrm{S}_{0}$ geometry was calculated at the B3LYP/6-31G(d) level of theory. The $\mathrm{S}_{1}$ and $\mathrm{T}_{1}$ geometries were calculated at the TD-B3LYP/6$31 \mathrm{G}(\mathrm{d})$ level of theory. The $\mathrm{T}_{3}$ geometry was calculated at the TD-B3LYP/6-31G(d) and UB3LYP/6-31G(d) levels of theory. 


\section{$\mathrm{S}_{0}$ geom $\quad \mathrm{S}_{1}$ geom $\quad \mathrm{T}_{1}$ geom $\quad \mathrm{T}_{3}$ geom}

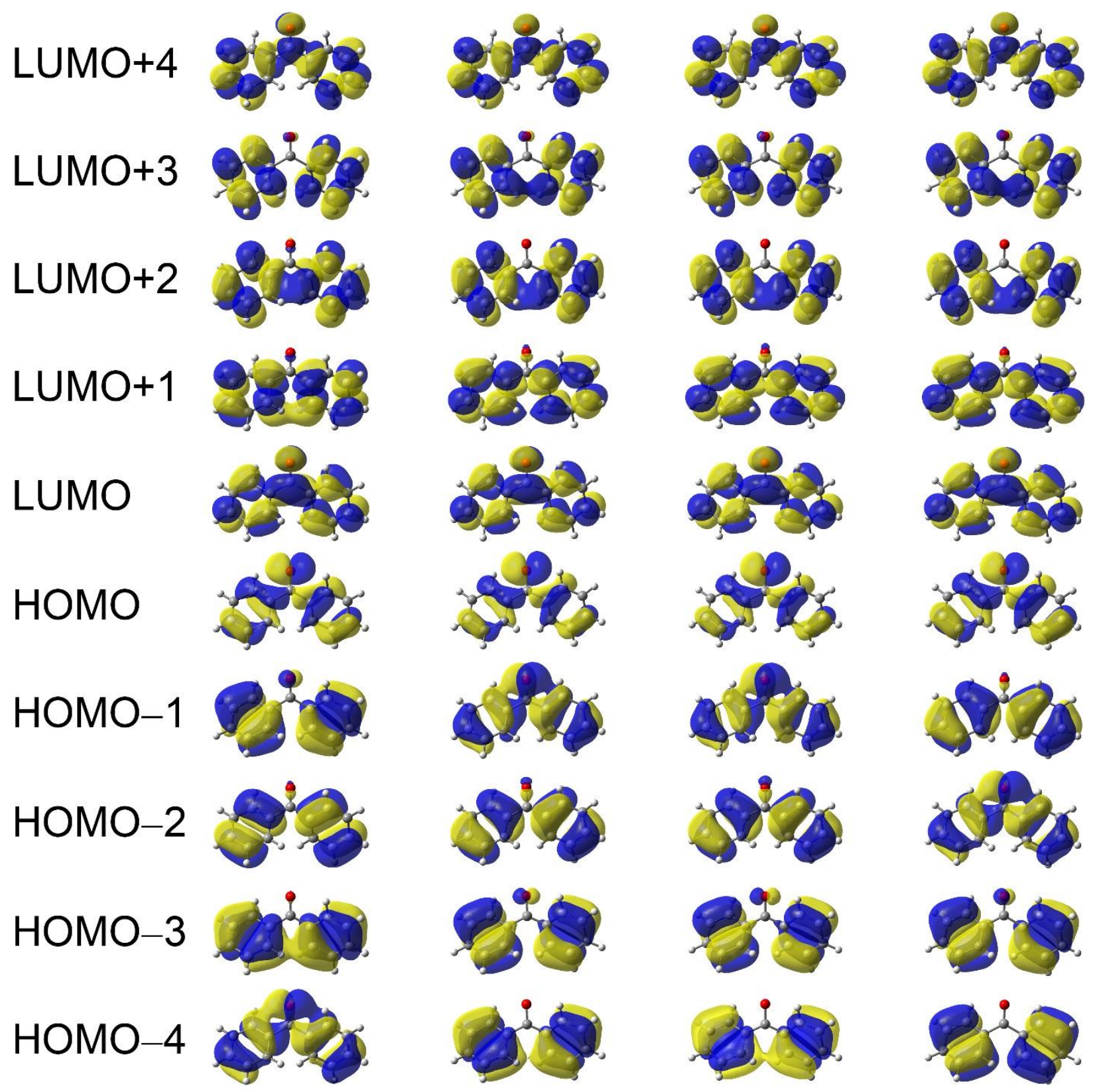

Figure S2. LUMO+4, LUMO+3, LUMO+2, LUMO+1, LUMO, HOMO, HOMO-1, HOMO-2, HOMO-3, and HOMO-4 of BP calculated for the $\mathrm{S}_{0}, \mathrm{~S}_{1}, \mathrm{~T}_{1}$, and $\mathrm{T}_{3}$ geometries at the B3LYP/6$31 \mathrm{G}(\mathrm{d})$ level of theory. 
Table S6. $\mathrm{S}_{1}, \mathrm{~T}_{1}, \mathrm{~T}_{2}$, and $\mathrm{T}_{3}$ of BP calculated at the $\mathrm{S}_{0}$ geometry. The calculated $\mathrm{S}_{0}-\mathrm{S}_{1}$ transition dipole moment is (0.0000 0.0000-0.1002) in atomic unit. HOMO-4, HOMO-3, HOMO-2, HOMO-1, HOMO, LUMO, LUMO+1, LUMO+2, LUMO+3, and LUMO+4 are shown in Figure S2.

\begin{tabular}{|c|c|c|c|c|}
\hline $\begin{array}{l}\text { Excited } \\
\text { state }\end{array}$ & $\begin{array}{l}\text { Excitation } \\
\text { energy }(\mathrm{eV})\end{array}$ & Orbitals & Contribution (\%) & Description \\
\hline \multirow[t]{3}{*}{$\mathrm{S}_{1}$} & 3.5663 & $\mathrm{HOMO} \rightarrow$ LUMO & 79.48 & $\mathrm{n} \pi^{*}$ \\
\hline & & $\mathrm{HOMO}-4 \rightarrow$ LUMO & 17.80 & $\pi \pi^{*}$ \\
\hline & & $\mathrm{HOMO} \rightarrow \mathrm{LUMO}+4$ & 1.59 & $\mathrm{n} \pi^{*}$ \\
\hline \multirow[t]{5}{*}{$\mathrm{T}_{1}$} & 2.9398 & $\mathrm{HOMO} \rightarrow \mathrm{LUMO}$ & 90.10 & $\mathrm{n} \pi^{*}$ \\
\hline & & $\mathrm{HOMO} \rightarrow \mathrm{LUMO}+4$ & 1.73 & $\mathrm{n} \pi^{*}$ \\
\hline & & $\mathrm{HOMO}-2 \rightarrow \mathrm{LUMO}+4$ & 1.70 & $\pi \pi^{*}$ \\
\hline & & HOMO-1 $\rightarrow$ LUMO & 1.42 & $\pi \pi^{*}$ \\
\hline & & $\mathrm{HOMO}-3 \rightarrow \mathrm{LUMO}+1$ & 1.07 & $\pi \pi^{*}$ \\
\hline \multirow[t]{7}{*}{$\mathrm{T}_{2}$} & 3.4248 & HOMO-4 $\rightarrow$ LUMO & 69.88 & $\pi \pi^{*}$ \\
\hline & & $\mathrm{HOMO}-2 \rightarrow \mathrm{LUMO}+2$ & 7.61 & $\pi \pi^{*}$ \\
\hline & & $\mathrm{HOMO}-3 \rightarrow \mathrm{LUMO}+1$ & 7.09 & $\pi \pi^{*}$ \\
\hline & & $\mathrm{HOMO}-1 \rightarrow \mathrm{LUMO}+3$ & 4.95 & $\pi \pi^{*}$ \\
\hline & & HOMO-1 $\rightarrow$ LUMO & 3.27 & $\pi \pi^{*}$ \\
\hline & & $\mathrm{HOMO}-2 \rightarrow \mathrm{LUMO}+1$ & 1.50 & $\pi \pi^{*}$ \\
\hline & & $\mathrm{HOMO} \rightarrow \mathrm{LUMO}+4$ & 1.38 & $\mathrm{n} \pi^{*}$ \\
\hline \multirow[t]{11}{*}{$\mathrm{T}_{3}$} & 3.4921 & HOMO-2 $\rightarrow$ LUMO & 47.27 & $\pi \pi^{*}$ \\
\hline & & HOMO-3 $\rightarrow$ LUMO & 10.86 & $\pi \pi^{*}$ \\
\hline & & $\mathrm{HOMO}-1 \rightarrow \mathrm{LUMO}+1$ & 8.59 & $\pi \pi^{*}$ \\
\hline & & $\mathrm{HOMO}-3 \rightarrow \mathrm{LUMO}+3$ & 6.49 & $\pi \pi^{*}$ \\
\hline & & $\mathrm{HOMO}-4 \rightarrow \mathrm{LUMO}+2$ & 6.27 & $\pi \pi^{*}$ \\
\hline & & $\mathrm{HOMO} \rightarrow \mathrm{LUMO}+2$ & 5.82 & $\mathrm{n} \pi^{*}$ \\
\hline & & $\mathrm{HOMO}-4 \rightarrow \mathrm{LUMO}+1$ & 3.34 & $\pi \pi^{*}$ \\
\hline & & $\mathrm{HOMO}-1 \rightarrow \mathrm{LUMO}+2$ & 2.72 & $\pi \pi^{*}$ \\
\hline & & $\mathrm{HOMO}-2 \rightarrow \mathrm{LUMO}+3$ & 2.69 & $\pi \pi^{*}$ \\
\hline & & $\mathrm{HOMO} \rightarrow \mathrm{LUMO}+1$ & 1.88 & $\mathrm{n} \pi^{*}$ \\
\hline & & $\mathrm{HOMO}-2 \rightarrow \mathrm{LUMO}+4$ & 1.30 & $\pi \pi^{*}$ \\
\hline
\end{tabular}


Table S7. $\mathrm{S}_{1}, \mathrm{~T}_{1}, \mathrm{~T}_{2}$, and $\mathrm{T}_{3}$ of BP calculated at the $\mathrm{S}_{1}$ geometry. The calculated $\mathrm{S}_{0}-\mathrm{S}_{1}$ transition dipole moment is (0 $0-0.0851)$ in atomic unit. HOMO-4, HOMO-3, HOMO-2, HOMO-1, HOMO, LUMO, LUMO+1, LUMO+2, LUMO+3, and LUMO+4 are shown in Figure S2.

\begin{tabular}{|c|c|c|c|c|}
\hline $\begin{array}{l}\text { Excited } \\
\text { state }\end{array}$ & $\begin{array}{l}\text { Excitation } \\
\text { energy }(\mathrm{eV})\end{array}$ & Orbitals & Contribution (\%) & Description \\
\hline \multirow[t]{3}{*}{$\mathrm{S}_{1}$} & 2.8512 & $\mathrm{HOMO} \rightarrow$ LUMO & 83.22 & $\mathrm{n} \pi^{*}$ \\
\hline & & HOMO-1 $\rightarrow$ LUMO & 14.92 & $\pi \pi^{*}$ \\
\hline & & $\mathrm{HOMO} \rightarrow \mathrm{LUMO}+4$ & 1.20 & $\mathrm{n} \pi^{*}$ \\
\hline \multirow[t]{2}{*}{$\mathrm{T}_{1}$} & 2.1841 & $\mathrm{HOMO} \rightarrow \mathrm{LUMO}$ & 94.04 & $\mathrm{n} \pi^{*}$ \\
\hline & & $\mathrm{HOMO} \rightarrow \mathrm{LUMO}+4$ & 1.64 & $\mathrm{n} \pi^{*}$ \\
\hline \multirow[t]{3}{*}{$\mathrm{T}_{2}$} & 2.6606 & HOMO-1 $\rightarrow$ LUMO & 87.81 & $\pi \pi^{*}$ \\
\hline & & HOMO-5 $\rightarrow$ LUMO & 2.82 & $\pi \pi^{*}$ \\
\hline & & $\mathrm{HOMO}-2 \rightarrow \mathrm{LUMO}+1$ & 2.21 & $\pi \pi^{*}$ \\
\hline \multirow[t]{10}{*}{$\mathrm{T}_{3}$} & 3.2082 & HOMO-2 $\rightarrow$ LUMO & 71.05 & $\pi \pi^{*}$ \\
\hline & & $\mathrm{HOMO}-1 \rightarrow \mathrm{LUMO}+1$ & 6.65 & $\pi \pi^{*}$ \\
\hline & & $\mathrm{HOMO}-4 \rightarrow \mathrm{LUMO}+3$ & 4.27 & $\pi \pi^{*}$ \\
\hline & & $\mathrm{HOMO} \rightarrow \mathrm{LUMO}+1$ & 4.09 & $\mathrm{n} \pi^{*}$ \\
\hline & & $\mathrm{HOMO}-3 \rightarrow \mathrm{LUMO}+2$ & 4.01 & $\pi \pi^{*}$ \\
\hline & & $\mathrm{HOMO}-4 \rightarrow$ LUMO & 2.12 & $\pi \pi^{*}$ \\
\hline & & $\mathrm{HOMO} \rightarrow \mathrm{LUMO}+2$ & 1.68 & $\mathrm{n} \pi^{*}$ \\
\hline & & $\mathrm{HOMO}-1 \rightarrow \mathrm{LUMO}+2$ & 1.33 & $\pi \pi^{*}$ \\
\hline & & $\mathrm{HOMO}-2 \rightarrow \mathrm{LUMO}+4$ & 1.10 & $\pi \pi^{*}$ \\
\hline & & $\mathrm{HOMO}-3 \rightarrow \mathrm{LUMO}+1$ & 1.02 & $\pi \pi^{*}$ \\
\hline
\end{tabular}


Table S8. $\mathrm{S}_{1}, \mathrm{~T}_{1}, \mathrm{~T}_{2}$, and $\mathrm{T}_{3}$ of BP calculated at the $\mathrm{T}_{1}$ geometry. The calculated $\mathrm{S}_{0}-\mathrm{S}_{1}$ transition dipole moment is (0 $0-0.0965)$ in atomic unit. HOMO-4, HOMO-3, HOMO-2, HOMO-1, HOMO, LUMO, LUMO+1, LUMO+2, LUMO+3, and LUMO+4 are shown in Figure S2.

\begin{tabular}{|c|c|c|c|c|}
\hline $\begin{array}{l}\text { Excited } \\
\text { state }\end{array}$ & $\begin{array}{l}\text { Excitation } \\
\text { energy }(\mathrm{eV})\end{array}$ & Orbitals & Contribution (\%) & Description \\
\hline \multirow[t]{3}{*}{$\mathrm{S}_{1}$} & 2.7867 & $\mathrm{HOMO} \rightarrow$ LUMO & 82.73 & $\mathrm{n} \pi^{*}$ \\
\hline & & HOMO-1 $\rightarrow$ LUMO & 14.98 & $\pi \pi^{*}$ \\
\hline & & $\mathrm{HOMO} \rightarrow \mathrm{LUMO}+4$ & 1.22 & $\mathrm{n} \pi^{*}$ \\
\hline \multirow[t]{2}{*}{$\mathrm{T}_{1}$} & 2.0916 & $\mathrm{HOMO} \rightarrow \mathrm{LUMO}$ & 93.97 & $\mathrm{n} \pi^{*}$ \\
\hline & & $\mathrm{HOMO} \rightarrow \mathrm{LUMO}+4$ & 1.59 & $\mathrm{n} \pi^{*}$ \\
\hline \multirow[t]{3}{*}{$\mathrm{T}_{2}$} & 2.6084 & HOMO-1 $\rightarrow$ LUMO & 87.91 & $\pi \pi^{*}$ \\
\hline & & HOMO-5 $\rightarrow$ LUMO & 3.08 & $\pi \pi^{*}$ \\
\hline & & $\mathrm{HOMO}-2 \rightarrow \mathrm{LUMO}+1$ & 2.16 & $\pi \pi^{*}$ \\
\hline \multirow[t]{8}{*}{$\mathrm{T}_{3}$} & 3.1746 & HOMO-2 $\rightarrow$ LUMO & 71.21 & $\pi \pi^{*}$ \\
\hline & & $\mathrm{HOMO}-1 \rightarrow \mathrm{LUMO}+1$ & 7.24 & $\pi \pi^{*}$ \\
\hline & & $\mathrm{HOMO} \rightarrow \mathrm{LUMO}+1$ & 5.34 & $\mathrm{n} \pi^{*}$ \\
\hline & & $\mathrm{HOMO}-3 \rightarrow \mathrm{LUMO}+2$ & 4.41 & $\pi \pi^{*}$ \\
\hline & & $\mathrm{HOMO}-4 \rightarrow \mathrm{LUMO}+3$ & 3.89 & $\pi \pi^{*}$ \\
\hline & & HOMO-4 $\rightarrow$ LUMO & 1.82 & $\pi \pi^{*}$ \\
\hline & & $\mathrm{HOMO} \rightarrow \mathrm{LUMO}+2$ & 1.43 & $\mathrm{n} \pi^{*}$ \\
\hline & & $\mathrm{HOMO}-2 \rightarrow \mathrm{LUMO}+4$ & 1.12 & $\pi \pi^{*}$ \\
\hline
\end{tabular}


Table S9. $\mathrm{S}_{1}, \mathrm{~T}_{1}, \mathrm{~T}_{2}$, and $\mathrm{T}_{3}$ of BP calculated at the $\mathrm{T}_{3}$ geometry. The calculated $\mathrm{S}_{0}-\mathrm{S}_{1}$ transition dipole moment is (0 $0-0.0902)$ in atomic unit. HOMO-4, HOMO-3, HOMO-2, HOMO-1, HOMO, LUMO, LUMO+1, LUMO+2, LUMO+3, and LUMO+4 are shown in Figure S2.

\begin{tabular}{|c|c|c|c|c|}
\hline $\begin{array}{l}\text { Excited } \\
\text { state }\end{array}$ & $\begin{array}{l}\text { Excitation } \\
\text { energy }(\mathrm{eV})\end{array}$ & Orbitals & Contribution (\%) & Description \\
\hline \multirow[t]{3}{*}{$\mathrm{S}_{1}$} & 3.2990 & $\mathrm{HOMO} \rightarrow \mathrm{LUMO}$ & 67.56 & $\mathrm{n} \pi^{*}$ \\
\hline & & HOMO-2 $\rightarrow$ LUMO & 30.49 & $\pi \pi^{*}$ \\
\hline & & $\mathrm{HOMO} \rightarrow \mathrm{LUMO}+4$ & 1.04 & $\mathrm{n} \pi^{*}$ \\
\hline \multirow[t]{3}{*}{$\overline{\mathrm{T}_{1}}$} & 2.6288 & $\mathrm{HOMO} \rightarrow \mathrm{LUMO}$ & 89.47 & $\mathrm{n} \pi^{*}$ \\
\hline & & $\mathrm{HOMO}-1 \rightarrow \mathrm{LUMO}+1$ & 4.54 & $\pi \pi^{*}$ \\
\hline & & HOMO-3 $\rightarrow$ LUMO & 1.06 & $\pi \pi^{*}$ \\
\hline \multirow[t]{6}{*}{$\mathrm{T}_{2}$} & 3.0239 & HOMO-2 $\rightarrow$ LUMO & 83.75 & $\pi \pi^{*}$ \\
\hline & & $\mathrm{HOMO}-1 \rightarrow \mathrm{LUMO}+1$ & 6.80 & $\pi \pi^{*}$ \\
\hline & & $\mathrm{HOMO}-3 \rightarrow \mathrm{LUMO}+3$ & 1.38 & $\pi \pi^{*}$ \\
\hline & & $\mathrm{HOMO}-4 \rightarrow \mathrm{LUMO}+2$ & 1.34 & $\pi \pi^{*}$ \\
\hline & & $\mathrm{HOMO} \rightarrow \mathrm{LUMO}+4$ & 1.30 & $\mathrm{n} \pi^{*}$ \\
\hline & & $\mathrm{HOMO} \rightarrow \mathrm{LUMO}$ & 1.07 & $\mathrm{n} \pi^{*}$ \\
\hline \multirow[t]{8}{*}{$\mathrm{T}_{3}$} & 3.0660 & HOMO-1 $\rightarrow$ LUMO & 73.27 & $\pi \pi^{*}$ \\
\hline & & $\mathrm{HOMO} \rightarrow \mathrm{LUMO}+1$ & 7.78 & $\mathrm{n} \pi^{*}$ \\
\hline & & $\mathrm{HOMO}-2 \rightarrow \mathrm{LUMO}+1$ & 6.49 & $\pi \pi^{*}$ \\
\hline & & $\mathrm{HOMO}-4 \rightarrow \mathrm{LUMO}+3$ & 3.52 & $\pi \pi^{*}$ \\
\hline & & $\mathrm{HOMO}-3 \rightarrow \mathrm{LUMO}+2$ & 2.99 & $\pi \pi^{*}$ \\
\hline & & $\mathrm{HOMO}-1 \rightarrow \mathrm{LUMO}+4$ & 1.27 & $\pi \pi^{*}$ \\
\hline & & $\mathrm{HOMO}-3 \rightarrow \mathrm{LUMO}+1$ & 1.05 & $\pi \pi^{*}$ \\
\hline & & $\mathrm{HOMO} \rightarrow \mathrm{LUMO}+2$ & 1.01 & $\mathrm{n} \pi^{*}$ \\
\hline
\end{tabular}


Table S10. The ground $\left(\mathrm{S}_{0}\right)$ and excited state $\left(\mathrm{S}_{n}\right.$ and $\left.\mathrm{T}_{n}, n=1,2,3,4,5,6\right)$ electronic energies $(\mathrm{eV})$ of BP calculated for the $\mathrm{S}_{0}, \mathrm{~S}_{1}, \mathrm{~T}_{1}, \mathrm{~T}_{3}$ and UB3LYP geometries. The zero point of the energies is set to be the $\mathrm{S}_{0}$ energy for the $\mathrm{S}_{0}$ geometry. The $\mathrm{S}_{0}$ energies were calculated at the B3LYP/6$31 \mathrm{G}(\mathrm{d})$ level of theory. The $\mathrm{S}_{0}-\mathrm{S}_{n}$ and $\mathrm{S}_{0}-\mathrm{T}_{n}$ excitation energies $(n=1,2,3,4,5,6)$ were calculated at the TD-B3LYP/6-31G(d) level of theory.

\begin{tabular}{llllll}
\hline State & $\mathrm{S}_{0}$ geom & $\mathrm{S}_{1}$ geom & $\mathrm{T}_{1}$ geom & $\mathrm{T}_{3}$ geom & UB3LYP geom \\
\hline $\mathrm{S}_{0}$ & 0.0000 & 0.3856 & 0.4642 & 0.2299 & 0.4295 \\
$\mathrm{~S}_{1}$ & 3.5663 & 3.2368 & 3.2509 & 3.5289 & 3.2490 \\
$\mathrm{~S}_{2}$ & 4.5855 & 4.6474 & 4.7181 & 4.5387 & 4.7003 \\
$\mathrm{~S}_{3}$ & 4.6497 & 4.6843 & 4.7382 & 4.6209 & 4.7248 \\
$\mathrm{~S}_{4}$ & 4.7583 & 4.7511 & 4.816 & 4.6942 & 4.8055 \\
$\mathrm{~S}_{5}$ & 4.9700 & 4.9647 & 4.9938 & 4.8683 & 4.9928 \\
$\mathrm{~S}_{6}$ & 5.3762 & 5.4228 & 5.4131 & 5.4183 & 5.4171 \\
\hline $\mathrm{T}_{1}$ & 2.9398 & 2.5697 & 2.5558 & 2.8587 & 2.5586 \\
$\mathrm{~T}_{2}$ & 3.4248 & 3.0462 & 3.0726 & 3.2538 & 3.0866 \\
$\mathrm{~T}_{3}$ & 3.4921 & 3.5938 & 3.6388 & 3.2959 & 3.6402 \\
$\mathrm{~T}_{4}$ & 4.0292 & 4.1071 & 4.1752 & 4.0685 & 4.1612 \\
$\mathrm{~T}_{5}$ & 4.0840 & 4.1453 & 4.2240 & 4.0953 & 4.2063 \\
$\mathrm{~T}_{6}$ & 4.3447 & 4.5257 & 4.5814 & 4.5108 & 4.5523 \\
\hline \hline
\end{tabular}


Table S11. The absolute values of the Franck-Condon and adiabatic energy differences between $\mathrm{S}_{0}, \mathrm{~S}_{1}, \mathrm{~T}_{1}, \mathrm{~T}_{2}$, and, $\mathrm{T}_{3}$ for $\mathrm{BP}$ (in eV). For calculating adiabatic energy differences, the energy minimum of $T_{2}$ was set to be the $T_{2}$ energy calculated at the $S_{1}$ geometry $(3.0462 \mathrm{eV}$ in Table $\mathrm{S} 10)$. This is because the $T_{2}$ energy is lowest at the $\mathrm{S}_{1}$ geometry.

\begin{tabular}{llllll}
\hline \hline & \multicolumn{2}{l}{ Franck-Condon } & Adiabatic \\
\cline { 2 - 4 } & $\mathrm{S}_{0}$ geom & $\mathrm{S}_{1}$ geom & $\mathrm{T}_{1}$ geom & $\mathrm{T}_{3}$ geom & \\
\hline $\mathrm{S}_{0}-\mathrm{S}_{1}$ & 3.5663 & 2.8512 & 2.7867 & 3.2990 & 3.2368 \\
$\mathrm{~S}_{0}-\mathrm{T}_{1}$ & 2.9398 & 2.1841 & 2.0916 & 2.6288 & 2.5558 \\
$\mathrm{~S}_{0}-\mathrm{T}_{2}$ & 3.4248 & 2.6606 & 2.6084 & 3.0239 & 3.0462 \\
$\mathrm{~S}_{0}-\mathrm{T}_{3}$ & 3.4921 & 3.2082 & 3.1746 & 3.0660 & 3.2959 \\
\hline $\mathrm{S}_{1}-\mathrm{T}_{1}$ & 0.6265 & 0.6671 & 0.6951 & 0.6702 & 0.681 \\
$\mathrm{~S}_{1}-\mathrm{T}_{2}$ & 0.1415 & 0.1906 & 0.1783 & 0.2751 & 0.1906 \\
$\mathrm{~S}_{1}-\mathrm{T}_{3}$ & 0.0742 & 0.3570 & 0.3879 & 0.2330 & 0.0591 \\
\hline $\mathrm{T}_{1}-\mathrm{T}_{2}$ & 0.4850 & 0.4765 & 0.5168 & 0.3951 & 0.4904 \\
$\mathrm{~T}_{1}-\mathrm{T}_{3}$ & 0.5523 & 1.0241 & 1.0830 & 0.4372 & 0.7401 \\
$\mathrm{~T}_{2}-\mathrm{T}_{3}$ & 0.0673 & 0.5476 & 0.5662 & 0.0421 & 0.2497 \\
\hline \hline
\end{tabular}




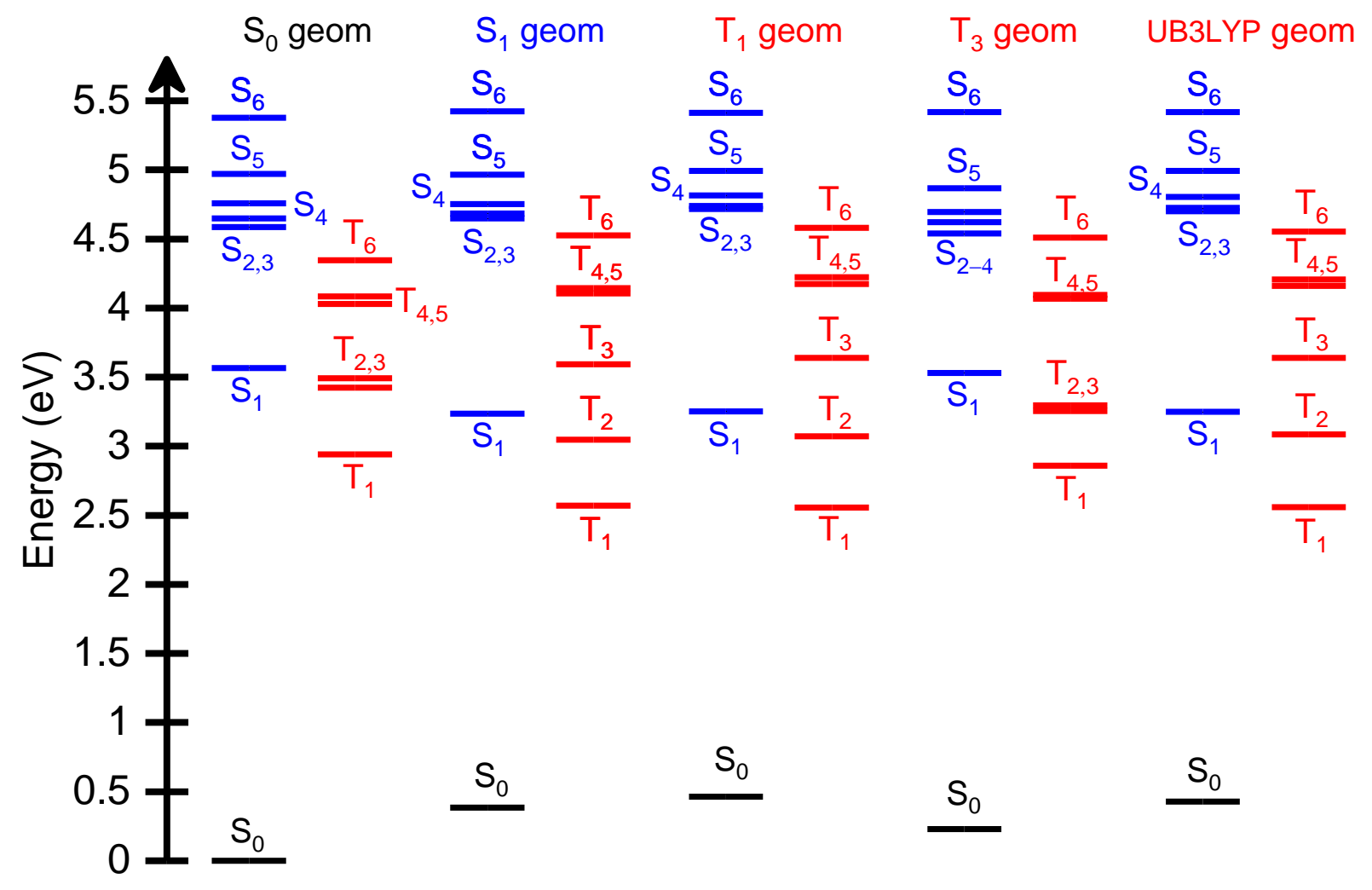

Figure S3. An energy-level diagram for BP. The numerical values of the electronic energies are listed in Table S10. The zero point of the electronic energies is set to be the $\mathrm{S}_{0}$ energy calculated at the optimized $\mathrm{S}_{0}$ geometry. 
Table S12. Absolute values of SOCs $\left(\mathrm{cm}^{-1}\right)$ calculated at the TD-B3LYP/6-31G(d) level of theory.

\begin{tabular}{|c|c|c|c|c|}
\hline & $\mathrm{S}_{0}$ & $\overline{S_{1}}$ & $\mathrm{~T}_{1}$ & $\mathrm{~T}_{3}$ \\
\hline$\left|\left\langle\mathrm{S}_{0}\left|\mathcal{H}_{\mathrm{SOC}}\right| \mathrm{T}_{1}^{0}\right\rangle\right|$ & 45.017 & 47.784 & 48.397 & 35.848 \\
\hline$\left|\left\langle\mathrm{S}_{0}\left|\mathcal{H}_{\text {SOC }}\right| \mathrm{T}_{1}^{1}\right\rangle\right|$ & 0.000 & 0.000 & 0.000 & 0.000 \\
\hline$\left|\left\langle\mathrm{S}_{0}\left|\mathcal{H}_{\text {SOC }}\right| \mathrm{T}_{1}^{-1}\right\rangle\right|$ & 0.000 & 0.000 & 0.000 & 0.000 \\
\hline$\sqrt{\sum_{M_{S}=0, \pm 1}\left|\left\langle\mathrm{~S}_{0}\left|\mathcal{H}_{\mathrm{SOC}}\right| \mathrm{T}_{1}^{M_{\mathrm{S}}}\right\rangle\right|^{2}}$ & 45.017 & 47.784 & 48.397 & 35.848 \\
\hline$\left|\left\langle\mathrm{S}_{1}\left|\mathcal{H}_{\mathrm{SOC}}\right| \mathrm{T}_{1}^{0}\right\rangle\right|$ & 9.105 & 14.609 & 15.142 & 14.839 \\
\hline$\left|\left\langle\mathrm{S}_{1}\left|\mathcal{H}_{\mathrm{SOC}}\right| \mathrm{T}_{1}^{1}\right\rangle\right|$ & 0.000 & 0.000 & 0.000 & 0.000 \\
\hline$\left|\left\langle\mathrm{S}_{1}\left|\mathcal{H}_{\mathrm{SOC}}\right| \mathrm{T}_{1}^{-1}\right\rangle\right|$ & 0.000 & 0.000 & 0.000 & 0.000 \\
\hline$\sqrt{\sum_{M_{\mathrm{S}}=0, \pm 1}\left|\left\langle\mathrm{~S}_{1}\left|\mathcal{H}_{\mathrm{SOC}}\right| \mathrm{T}_{1}^{M_{\mathrm{S}}}\right\rangle\right|^{2}}$ & 9.105 & 14.609 & 15.142 & 14.839 \\
\hline$\left|\left\langle\mathrm{S}_{1}\left|\mathcal{H}_{\mathrm{SOC}}\right| \mathrm{T}_{2}^{0}\right\rangle\right|$ & 18.041 & 28.122 & 28.178 & 18.505 \\
\hline$\left|\left\langle\mathrm{S}_{1}\left|\mathcal{H}_{\mathrm{SOC}}\right| \mathrm{T}_{2}^{1}\right\rangle\right|$ & 0.000 & 0.000 & 0.000 & 0.000 \\
\hline$\left|\left\langle\mathrm{S}_{1}\left|\mathcal{H}_{\mathrm{SOC}}\right| \mathrm{T}_{2}^{-1}\right\rangle\right|$ & 0.000 & 0.000 & 0.000 & 0.000 \\
\hline$\sqrt{\sum_{M_{\mathrm{S}}=0, \pm 1}\left|\left\langle\mathrm{~S}_{1}\left|\mathcal{H}_{\mathrm{SOC}}\right| \mathrm{T}_{2}^{M_{\mathrm{S}}}\right\rangle\right|^{2}}$ & 18.041 & 28.122 & 28.178 & 18.505 \\
\hline$\left|\left\langle\mathrm{S}_{1}\left|\mathcal{H}_{\mathrm{SOC}}\right| \mathrm{T}_{3}^{0}\right\rangle\right|$ & 0.000 & 0.000 & 0.000 & 0.000 \\
\hline$\left|\left\langle\mathrm{S}_{1}\left|\mathcal{H}_{\mathrm{SOC}}\right| \mathrm{T}_{3}^{1}\right\rangle\right|$ & 2.809 & 4.908 & 5.764 & 2.604 \\
\hline$\left|\left\langle\mathrm{S}_{1}\left|\mathcal{H}_{\mathrm{SOC}}\right| \mathrm{T}_{3}^{-1}\right\rangle\right|$ & 2.809 & 4.908 & 5.764 & 2.604 \\
\hline$\sqrt{\sum_{M_{\mathrm{S}}=0, \pm 1}\left|\left\langle\mathrm{~S}_{1}\left|\mathcal{H}_{\mathrm{SOC}}\right| \mathrm{T}_{3}^{M_{\mathrm{S}}}\right\rangle\right|^{2}}$ & 3.973 & 6.941 & 8.151 & 3.683 \\
\hline
\end{tabular}




\section{The kinetic equations for calculating $S_{0}, S_{1}, T_{1}, T_{2}$, and $T_{3}$ populations}

$$
\begin{aligned}
& \frac{d}{d t}\left[\mathrm{~S}_{1} @ \mathrm{~S}_{0}\right](t)= \\
& -\left(k_{\mathrm{S}_{1} \rightarrow \mathrm{T}_{1}}^{\mathrm{S}_{0}}+k_{\mathrm{S}_{1} \rightarrow \mathrm{T}_{2}}^{\mathrm{S}_{0}}+k_{\mathrm{S}_{1} \rightarrow \mathrm{T}_{3}}^{\mathrm{S}_{0}}+k_{\mathrm{S}_{1} \rightarrow \mathrm{S}_{0}}^{\mathrm{S}_{0}}+k_{\mathrm{F}}^{\mathrm{S}_{0}}+k_{\mathrm{S}_{1}, \mathrm{GR}}\right)\left[\mathrm{S}_{1} @ \mathrm{~S}_{0}\right](t) \\
& +k_{\mathrm{T}_{1} \rightarrow \mathrm{S}_{1}}^{\mathrm{S}_{0}}\left[\mathrm{~T}_{1} @ \mathrm{~S}_{0}\right](t)+k_{\mathrm{T}_{2} \rightarrow \mathrm{S}_{1}}^{\mathrm{S}_{0}}\left[\mathrm{~T}_{2} @ \mathrm{~S}_{0}\right](t)+k_{\mathrm{T}_{3} \rightarrow \mathrm{S}_{1}}^{\mathrm{S}_{0}}\left[\mathrm{~T}_{3} @ \mathrm{~S}_{0}\right](t)
\end{aligned}
$$

$$
\begin{aligned}
& \frac{d}{d t}\left[\mathrm{~T}_{1} @ \mathrm{~S}_{0}\right](t)= \\
& -\left(k_{\mathrm{T}_{1} \rightarrow \mathrm{S}_{1}}^{\mathrm{S}_{0}}+k_{\mathrm{T}_{1} \rightarrow \mathrm{T}_{2}}^{\mathrm{S}_{0}}+k_{\mathrm{T}_{1} \rightarrow \mathrm{T}_{3}}^{\mathrm{S}_{0}}+k_{\mathrm{T}_{1} \rightarrow \mathrm{S}_{0}}^{\mathrm{S}_{0}}+k_{\mathrm{P}}^{\mathrm{S}_{0}}+k_{\mathrm{T}_{1}, \mathrm{GR}}\right)\left[\mathrm{T}_{1} @ \mathrm{~S}_{0}\right](t) \\
& +k_{\mathrm{S}_{1} \rightarrow \mathrm{T}_{1}}^{\mathrm{S}_{0}}\left[\mathrm{~S}_{1} @ \mathrm{~S}_{0}\right](t)+k_{\mathrm{T}_{2} \rightarrow \mathrm{T}_{1}}^{\mathrm{S}_{0}}\left[\mathrm{~T}_{2} @ \mathrm{~S}_{0}\right](t)+k_{\mathrm{T}_{3} \rightarrow \mathrm{T}_{1}}^{\mathrm{S}_{0}}\left[\mathrm{~T}_{3} @ \mathrm{~S}_{0}\right](t)
\end{aligned}
$$

$$
\begin{aligned}
& \frac{d}{d t}\left[\mathrm{~T}_{2} @ \mathrm{~S}_{0}\right](t)= \\
& -\left(k_{\mathrm{T}_{2} \rightarrow \mathrm{S}_{1}}^{\mathrm{S}_{0}}+k_{\mathrm{T}_{2} \rightarrow \mathrm{T}_{1}}^{\mathrm{S}_{0}}+k_{\mathrm{T}_{2} \rightarrow \mathrm{T}_{3}}^{\mathrm{S}_{0}}+k_{\mathrm{T}_{2}, \mathrm{GR}}\right)\left[\mathrm{T}_{2} @ \mathrm{~S}_{0}\right](t) \\
& +k_{\mathrm{S}_{1} \rightarrow \mathrm{T}_{2}}^{\mathrm{S}_{0}}\left[\mathrm{~S}_{1} @ \mathrm{~S}_{0}\right](t)+k_{\mathrm{T}_{1} \rightarrow \mathrm{T}_{2}}^{\mathrm{S}_{0}}\left[\mathrm{~T}_{1} @ \mathrm{~S}_{0}\right](t)+k_{\mathrm{T}_{3} \rightarrow \mathrm{T}_{2}}^{\mathrm{S}_{0}}\left[\mathrm{~T}_{3} @ \mathrm{~S}_{0}\right](t)
\end{aligned}
$$

$$
\frac{d}{d t}\left[\mathrm{~T}_{3} @ \mathrm{~S}_{0}\right](t)=
$$$$
-\left(k_{\mathrm{T}_{3} \rightarrow \mathrm{S}_{1}}^{\mathrm{S}_{0}}+k_{\mathrm{T}_{3} \rightarrow \mathrm{T}_{2}}^{\mathrm{S}_{0}}+k_{\mathrm{T}_{3} \rightarrow \mathrm{T}_{1}}^{\mathrm{S}_{0}}+k_{\mathrm{T}_{3}, \mathrm{GR}}\right)\left[\mathrm{T}_{3} @ \mathrm{~S}_{0}\right](t)
$$$$
+k_{\mathrm{S}_{1} \rightarrow \mathrm{T}_{3}}^{\mathrm{S}_{3}}\left[\mathrm{~S}_{1} @ \mathrm{~S}_{0}\right](t)+k_{\mathrm{T}_{2} \rightarrow \mathrm{T}_{3}}^{\mathrm{S}_{0}}\left[\mathrm{~T}_{2} @ \mathrm{~S}_{0}\right](t)+k_{\mathrm{T}_{1} \rightarrow \mathrm{T}_{3}}^{\mathrm{S}_{0}}\left[\mathrm{~T}_{1} @ \mathrm{~S}_{0}\right](t)
$$

$$
\begin{aligned}
& \frac{d}{d t}\left[\mathrm{~S}_{0} @ \mathrm{~S}_{0}\right](t)= \\
& +\left(k_{\mathrm{S}_{1} \rightarrow \mathrm{S}_{0}}^{\mathrm{S}_{0}}+k_{\mathrm{F}}^{\mathrm{S}_{0}}\right)\left[\mathrm{S}_{1} @ \mathrm{~S}_{0}\right](t)+\left(k_{\mathrm{T}_{1} \rightarrow \mathrm{S}_{0}}^{\mathrm{S}_{0}}+k_{\mathrm{P}}^{\mathrm{S}_{0}}\right)\left[\mathrm{T}_{1} @ \mathrm{~S}_{0}\right](t) \\
& +k_{\mathrm{S}_{0}, \mathrm{GR}}\left\{\left[\mathrm{S}_{0} @ \mathrm{~S}_{1}\right](t)+\left[\mathrm{S}_{0} @ \mathrm{~T}_{1}\right](t)+\left[\mathrm{S}_{0} @ \mathrm{~T}_{3}\right](t)\right\}
\end{aligned}
$$




$$
\begin{aligned}
& \frac{d}{d t}\left[\mathrm{~S}_{1} @ \mathrm{~S}_{1}\right](t)= \\
& -\left(k_{\mathrm{S}_{1} \rightarrow \mathrm{T}_{1}}^{\mathrm{S}_{1}}+k_{\mathrm{S}_{1} \rightarrow \mathrm{T}_{2}}^{\mathrm{S}_{1}}+k_{\mathrm{S}_{1} \rightarrow \mathrm{T}_{3}}^{\mathrm{S}_{1}}+k_{\mathrm{S}_{1} \rightarrow \mathrm{S}_{0}}^{\mathrm{S}_{1}}+k_{\mathrm{F}}^{\mathrm{S}_{1}}+k_{\mathrm{S}_{1}, \mathrm{GR}}\right)\left[\mathrm{S}_{1} @ \mathrm{~S}_{1}\right](t) \\
& +k_{\mathrm{T}_{1} \rightarrow \mathrm{S}_{1}}^{\mathrm{S}_{1}}\left[\mathrm{~T}_{1} @ \mathrm{~S}_{1}\right](t)+k_{\mathrm{T}_{2} \rightarrow \mathrm{S}_{1}}^{\mathrm{S}_{1}}\left[\mathrm{~T}_{2} @ \mathrm{~S}_{1}\right](t)+k_{\mathrm{T}_{3} \rightarrow \mathrm{S}_{1}}^{\mathrm{S}_{1}}\left[\mathrm{~T}_{3} @ \mathrm{~S}_{1}\right](t)+k_{\mathrm{S}_{1}, \mathrm{GR}}\left[\mathrm{S}_{1} @ \mathrm{~S}_{0}\right](t) \\
& \frac{d}{d t}\left[\mathrm{~T}_{1} @ \mathrm{~S}_{1}\right](t)= \\
& -\left(k_{\mathrm{T}_{1} \rightarrow \mathrm{S}_{1}}^{\mathrm{S}_{1}}+k_{\mathrm{T}_{1} \rightarrow \mathrm{T}_{2}}^{\mathrm{S}_{1}}+k_{\mathrm{T}_{1} \rightarrow \mathrm{T}_{3}}^{\mathrm{S}_{1}}+k_{\mathrm{T}_{1} \rightarrow \mathrm{S}_{0}}^{\mathrm{S}_{1}}+k_{\mathrm{P}}^{\mathrm{S}_{1}}+k_{\mathrm{T}_{1}, \mathrm{GR}}\right)\left[\mathrm{T}_{1} @ \mathrm{~S}_{1}\right](t) \\
& +k_{\mathrm{S}_{1} \rightarrow \mathrm{T}_{1}}^{\mathrm{S}_{1}}\left[\mathrm{~S}_{1} @ \mathrm{~S}_{1}\right](t)+k_{\mathrm{T}_{2} \rightarrow \mathrm{T}_{1}}^{\mathrm{S}_{1}}\left[\mathrm{~T}_{2} @ \mathrm{~S}_{1}\right](t)+k_{\mathrm{T}_{3} \rightarrow \mathrm{T}_{1}}^{\mathrm{S}_{1}}\left[\mathrm{~T}_{3} @ \mathrm{~S}_{1}\right](t) \\
& \frac{d}{d t}\left[\mathrm{~T}_{2} @ \mathrm{~S}_{1}\right](t)= \\
& -\left(k_{\mathrm{T}_{2} \rightarrow \mathrm{S}_{1}}^{\mathrm{S}_{1}}++k_{\mathrm{T}_{2} \rightarrow \mathrm{T}_{1}}^{\mathrm{S}_{1}}+k_{\mathrm{T}_{2} \rightarrow \mathrm{T}_{3}}^{\mathrm{S}_{1}}+k_{\mathrm{T}_{2}, \mathrm{GR}}\right)\left[\mathrm{T}_{2} @ \mathrm{~S}_{1}\right](t) \\
& +k_{\mathrm{S}_{1} \rightarrow \mathrm{T}_{2}}^{\mathrm{S}_{1}}\left[\mathrm{~S}_{1} @ \mathrm{~S}_{1}\right](t)+k_{\mathrm{T}_{1} \rightarrow \mathrm{T}_{2}}^{\mathrm{S}_{1}}\left[\mathrm{~T}_{1} @ \mathrm{~S}_{1}\right](t)+k_{\mathrm{T}_{3} \rightarrow \mathrm{T}_{2}}^{\mathrm{S}_{1}}\left[\mathrm{~T}_{1} @ \mathrm{~S}_{1}\right](t) \\
& \frac{d}{d t}\left[\mathrm{~T}_{3} @ \mathrm{~S}_{1}\right](t)= \\
& -\left(k_{\mathrm{T}_{3} \rightarrow \mathrm{S}_{1}}^{\mathrm{S}_{1}}+k_{\mathrm{T}_{3} \rightarrow \mathrm{T}_{2}}^{\mathrm{S}_{1}}+k_{\mathrm{T}_{3} \rightarrow \mathrm{T}_{1}}^{\mathrm{S}_{1}}+k_{\mathrm{T}_{3}, \mathrm{GR}}\right)\left[\mathrm{T}_{3} @ \mathrm{~S}_{1}\right](t) \\
& +k_{\mathrm{S}_{1} \rightarrow \mathrm{T}_{3}}^{\mathrm{S}_{1}}\left[\mathrm{~S}_{1} @ \mathrm{~S}_{1}\right](t)+k_{\mathrm{T}_{2} \rightarrow \mathrm{T}_{3}}^{\mathrm{S}_{1}}\left[\mathrm{~T}_{2} @ \mathrm{~S}_{1}\right](t)+k_{\mathrm{T}_{1} \rightarrow \mathrm{T}_{3}}^{\mathrm{S}_{1}}\left[\mathrm{~T}_{1} @ \mathrm{~S}_{1}\right](t) \\
& \frac{d}{d t}\left[\mathrm{~S}_{0} @ \mathrm{~S}_{1}\right](t)= \\
& -k_{\mathrm{S}_{0}, \mathrm{GR}}\left[\mathrm{S}_{0} @ \mathrm{~S}_{1}\right](t)+\left(k_{\mathrm{S}_{1} \rightarrow \mathrm{S}_{0}}^{\mathrm{S}_{1}}+k_{\mathrm{F}}^{\mathrm{S}_{1}}\right)\left[\mathrm{S}_{1} @ \mathrm{~S}_{1}\right](t)+\left(k_{\mathrm{T}_{1} \rightarrow \mathrm{S}_{0}}^{\mathrm{S}_{1}}+k_{\mathrm{P}}^{\mathrm{S}_{1}}\right)\left[\mathrm{T}_{1} @ \mathrm{~S}_{1}\right](t)
\end{aligned}
$$




$$
\begin{aligned}
& \frac{d}{d t}\left[\mathrm{~S}_{1} @ \mathrm{~T}_{1}\right](t)= \\
& -\left(k_{\mathrm{S}_{1} \rightarrow \mathrm{T}_{1}}^{\mathrm{T}_{1}}+k_{\mathrm{S}_{1} \rightarrow \mathrm{T}_{2}}^{\mathrm{T}_{1}}+k_{\mathrm{S}_{1} \rightarrow \mathrm{T}_{3}}^{\mathrm{T}_{1}}+k_{\mathrm{S}_{1} \rightarrow \mathrm{S}_{0}}^{\mathrm{T}_{1}}+k_{\mathrm{F}}^{\mathrm{T}_{1}}+k_{\mathrm{S}_{1}, \mathrm{GR}}\right)\left[\mathrm{S}_{1} @ \mathrm{~T}_{1}\right](t) \\
& +k_{\mathrm{T}_{1} \rightarrow \mathrm{S}_{1}}^{\mathrm{T}_{1}}\left[\mathrm{~T}_{1} @ \mathrm{~T}_{1}\right](t)+k_{\mathrm{T}_{2} \rightarrow \mathrm{S}_{1}}^{\mathrm{T}_{1}}\left[\mathrm{~T}_{2} @ \mathrm{~T}_{1}\right](t)+k_{\mathrm{T}_{3} \rightarrow \mathrm{S}_{1}}^{\mathrm{T}_{1}}\left[\mathrm{~T}_{3} @ \mathrm{~T}_{1}\right](t)
\end{aligned}
$$

$$
\begin{aligned}
& \frac{d}{d t}\left[\mathrm{~T}_{1} @ \mathrm{~T}_{1}\right](t)= \\
& -\left(k_{\mathrm{T}_{1} \rightarrow \mathrm{S}_{1}}^{\mathrm{T}_{1}}+k_{\mathrm{T}_{1} \rightarrow \mathrm{T}_{2}}^{\mathrm{T}_{1}}+k_{\mathrm{T}_{1} \rightarrow \mathrm{T}_{3}}^{\mathrm{T}_{1}}+k_{\mathrm{T}_{1} \rightarrow \mathrm{S}_{0}}^{\mathrm{T}_{1}}+k_{\mathrm{P}}^{\mathrm{T}_{1}}\right)\left[\mathrm{T}_{1} @ \mathrm{~T}_{1}\right](t) \\
& +k_{\mathrm{S}_{1} \rightarrow \mathrm{T}_{1}}^{\mathrm{T}_{1}}\left[\mathrm{~S}_{1} @ \mathrm{~T}_{1}\right](t)+k_{\mathrm{T}_{2} \rightarrow \mathrm{T}_{1}}^{\mathrm{T}_{1}}\left[\mathrm{~T}_{2} @ \mathrm{~T}_{1}\right](t)+k_{\mathrm{T}_{3} \rightarrow \mathrm{T}_{1}}^{\mathrm{T}_{1}}\left[\mathrm{~T}_{3} @ \mathrm{~T}_{1}\right](t) \\
& +k_{\mathrm{T}_{1}, \mathrm{GR}}\left\{\left[\mathrm{T}_{1} @ \mathrm{~S}_{0}\right](t)+\left[\mathrm{T}_{1} @ \mathrm{~S}_{1}\right](t)+\left[\mathrm{T}_{1} @ \mathrm{~S}_{3}\right](t)\right\} \\
& +k_{\mathrm{T}_{2}, \mathrm{GR}}\left\{\left[\mathrm{T}_{2} @ \mathrm{~S}_{0}\right](t)+\left[\mathrm{T}_{2} @ \mathrm{~S}_{1}\right](t)+\left[\mathrm{T}_{2} @ \mathrm{~S}_{3}\right](t)\right\}
\end{aligned}
$$

$$
\begin{aligned}
& \frac{d}{d t}\left[\mathrm{~T}_{2} @ \mathrm{~T}_{1}\right](t)= \\
& -\left(k_{\mathrm{T}_{2} \rightarrow \mathrm{S}_{1}}^{\mathrm{T}_{1}}+k_{\mathrm{T}_{2} \rightarrow \mathrm{T}_{1}}^{\mathrm{T}_{1}}+k_{\mathrm{T}_{2} \rightarrow \mathrm{T}_{3}}^{\mathrm{T}_{1}}\right)\left[\mathrm{T}_{2} @ \mathrm{~T}_{1}\right](t) \\
& +k_{\mathrm{S}_{1} \rightarrow \mathrm{T}_{2}}^{\mathrm{T}_{1}}\left[\mathrm{~S}_{1} @ \mathrm{~T}_{1}\right](t)+k_{\mathrm{T}_{1} \rightarrow \mathrm{T}_{2}}^{\mathrm{T}_{1}}\left[\mathrm{~T}_{1} @ \mathrm{~T}_{1}\right](t)+k_{\mathrm{T}_{3} \rightarrow \mathrm{T}_{2}}^{\mathrm{T}_{1}}\left[\mathrm{~T}_{3} @ \mathrm{~T}_{1}\right](t)
\end{aligned}
$$

$$
\begin{aligned}
& \frac{d}{d t}\left[\mathrm{~T}_{3} @ \mathrm{~T}_{1}\right](t)= \\
& -\left(k_{\mathrm{T}_{3} \rightarrow \mathrm{S}_{1}}^{\mathrm{T}_{1}}+k_{\mathrm{T}_{3} \rightarrow \mathrm{T}_{2}}^{\mathrm{T}_{1}}+k_{\mathrm{T}_{3} \rightarrow \mathrm{T}_{1}}^{\mathrm{T}_{1}}+k_{\mathrm{T}_{3}, \mathrm{GR}}\right)\left[\mathrm{T}_{3} @ \mathrm{~T}_{1}\right](t) \\
& +k_{\mathrm{S}_{1} \rightarrow \mathrm{T}_{3}}^{\mathrm{T}_{1}}\left[\mathrm{~S}_{1} @ \mathrm{~T}_{1}\right](t)+k_{\mathrm{T}_{2} \rightarrow \mathrm{T}_{3}}^{\mathrm{T}_{1}}\left[\mathrm{~T}_{2} @ \mathrm{~T}_{1}\right](t)+k_{\mathrm{T}_{1} \rightarrow \mathrm{T}_{3}}^{\mathrm{T}_{1}}\left[\mathrm{~T}_{1} @ \mathrm{~T}_{1}\right](t)
\end{aligned}
$$

$$
\begin{aligned}
& \frac{d}{d t}\left[\mathrm{~S}_{0} @ \mathrm{~T}_{1}\right](t)= \\
& -k_{\mathrm{S}_{0}, \mathrm{GR}}\left[\mathrm{S}_{0} @ \mathrm{~T}_{1}\right](t)+\left(k_{\mathrm{S}_{1} \rightarrow \mathrm{S}_{0}}^{\mathrm{T}_{1}}+k_{\mathrm{F}}^{\mathrm{T}_{1}}\right)\left[\mathrm{S}_{1} @ \mathrm{~T}_{1}\right](t)+\left(k_{\mathrm{T}_{1} \rightarrow \mathrm{S}_{0}}^{\mathrm{T}_{1}}+k_{\mathrm{P}}^{\mathrm{T}_{1}}\right)\left[\mathrm{T}_{1} @ \mathrm{~T}_{1}\right](t)
\end{aligned}
$$




$$
\begin{aligned}
& \frac{d}{d t}\left[\mathrm{~S}_{1} @ \mathrm{~T}_{3}\right](t)= \\
& -\left(k_{\mathrm{S}_{1} \rightarrow \mathrm{T}_{1}}^{\mathrm{T}_{3}}+k_{\mathrm{S}_{1} \rightarrow \mathrm{T}_{2}}^{\mathrm{T}_{3}}+k_{\mathrm{S}_{1} \rightarrow \mathrm{T}_{3}}^{\mathrm{T}_{3}}+k_{\mathrm{S}_{1} \rightarrow \mathrm{S}_{0}}^{\mathrm{T}_{3}}+k_{\mathrm{F}}^{\mathrm{T}_{3}}+k_{\mathrm{S}_{1}, \mathrm{GR}}\right)\left[\mathrm{S}_{1} @ \mathrm{~T}_{3}\right](t) \\
& +k_{\mathrm{T}_{1} \rightarrow \mathrm{S}_{1}}^{\mathrm{T}_{3}}\left[\mathrm{~T}_{1} @ \mathrm{~T}_{3}\right](t)+k_{\mathrm{T}_{2} \rightarrow \mathrm{S}_{1}}^{\mathrm{T}_{3}}\left[\mathrm{~T}_{2} @ \mathrm{~T}_{3}\right](t)+k_{\mathrm{T}_{3} \rightarrow \mathrm{S}_{1}}^{\mathrm{T}_{3}}\left[\mathrm{~T}_{3} @ \mathrm{~T}_{3}\right](t)
\end{aligned}
$$

$$
\begin{aligned}
& \frac{d}{d t}\left[\mathrm{~T}_{1} @ \mathrm{~T}_{3}\right](t)= \\
& -\left(k_{\mathrm{T}_{1} \rightarrow \mathrm{S}_{1}}^{\mathrm{T}_{3}}+k_{\mathrm{T}_{1} \rightarrow \mathrm{T}_{2}}^{\mathrm{T}_{3}}+k_{\mathrm{T}_{1} \rightarrow \mathrm{T}_{3}}^{\mathrm{T}_{3}}+k_{\mathrm{T}_{1} \rightarrow \mathrm{S}_{0}}^{\mathrm{T}_{3}}+k_{\mathrm{P}}^{\mathrm{T}_{3}}+k_{\mathrm{T}_{1}, \mathrm{GR}}\right)\left[\mathrm{T}_{1} @ \mathrm{~T}_{3}\right](t) \\
& +k_{\mathrm{S}_{1} \rightarrow \mathrm{T}_{1}}^{\mathrm{T}_{3}}\left[\mathrm{~S}_{1} @ \mathrm{~T}_{3}\right](t)+k_{\mathrm{T}_{2} \rightarrow \mathrm{T}_{1}}^{\mathrm{T}_{3}}\left[\mathrm{~T}_{2} @ \mathrm{~T}_{3}\right](t)+k_{\mathrm{T}_{3} \rightarrow \mathrm{T}_{1}}^{\mathrm{T}_{3}}\left[\mathrm{~T}_{3} @ \mathrm{~T}_{3}\right](t)
\end{aligned}
$$

$$
\frac{d}{d t}\left[\mathrm{~T}_{2} @ \mathrm{~T}_{3}\right](t)=
$$$$
-\left(k_{\mathrm{T}_{2} \rightarrow \mathrm{S}_{1}}^{\mathrm{T}_{3}}+k_{\mathrm{T}_{2} \rightarrow \mathrm{T}_{1}}^{\mathrm{T}_{3}}+k_{\mathrm{T}_{2} \rightarrow \mathrm{T}_{3}}^{\mathrm{T}_{3}}+k_{\mathrm{T}_{2}, \mathrm{GR}}\right)\left[\mathrm{T}_{2} @ \mathrm{~T}_{3}\right](t)
$$$$
+k_{\mathrm{S}_{1} \rightarrow \mathrm{T}_{2}}^{\mathrm{T}_{3}}\left[\mathrm{~S}_{1} @ \mathrm{~T}_{3}\right](t)+k_{\mathrm{T}_{1} \rightarrow \mathrm{T}_{2}}^{\mathrm{T}_{3}}\left[\mathrm{~T}_{1} @ \mathrm{~T}_{3}\right](t)+k_{\mathrm{T}_{3} \rightarrow \mathrm{T}_{2}}^{\mathrm{T}_{3}}\left[\mathrm{~T}_{3} @ \mathrm{~T}_{3}\right](t)
$$

$$
\begin{aligned}
& \frac{d}{d t}\left[\mathrm{~T}_{3} @ \mathrm{~T}_{3}\right](t)= \\
& -\left(k_{\mathrm{T}_{3} \rightarrow \mathrm{S}_{1}}^{\mathrm{T}_{3}}+k_{\mathrm{T}_{3} \rightarrow \mathrm{T}_{2}}^{\mathrm{T}_{3}}+k_{\mathrm{T}_{3} \rightarrow \mathrm{T}_{1}}^{\mathrm{T}_{3}}\right)\left[\mathrm{T}_{3} @ \mathrm{~T}_{3}\right](t) \\
& +k_{\mathrm{S}_{1} \rightarrow \mathrm{T}_{3}}^{\mathrm{T}_{3}}\left[\mathrm{~S}_{1} @ \mathrm{~T}_{3}\right](t)+k_{\mathrm{T}_{2} \rightarrow \mathrm{T}_{3}}^{\mathrm{T}_{3}}\left[\mathrm{~T}_{2} @ \mathrm{~T}_{3}\right](t)+k_{\mathrm{T}_{1} \rightarrow \mathrm{T}_{3}}^{\mathrm{T}_{3}}\left[\mathrm{~T}_{1} @ \mathrm{~T}_{3}\right](t) \\
& +k_{\mathrm{T}_{3}, \mathrm{GR}}\left\{\left[\mathrm{T}_{3} @ \mathrm{~S}_{0}\right](t)+\left[\mathrm{T}_{3} @ \mathrm{~S}_{1}\right](t)+\left[\mathrm{T}_{3} @ \mathrm{~T}_{1}\right](t)\right\}
\end{aligned}
$$

$$
\begin{aligned}
& \frac{d}{d t}\left[\mathrm{~S}_{0} @ \mathrm{~T}_{3}\right](t)= \\
& -k_{\mathrm{S}_{0}, \mathrm{GR}}\left[\mathrm{S}_{0} @ \mathrm{~T}_{3}\right](t)+\left(k_{\mathrm{S}_{1} \rightarrow \mathrm{S}_{0}}^{\mathrm{T}_{3}}+k_{\mathrm{F}}^{\mathrm{T}_{3}}\right)\left[\mathrm{S}_{1} @ \mathrm{~T}_{3}\right](t)+\left(k_{\mathrm{T}_{1} \rightarrow \mathrm{S}_{0}}^{\mathrm{T}_{3}}+k_{\mathrm{P}}^{\mathrm{T}_{3}}\right)\left[\mathrm{T}_{1} @ \mathrm{~T}_{3}\right](t)
\end{aligned}
$$


Table S13. Definitions of the variables and constants in Eqs S1-20.

\begin{tabular}{ll}
\hline$\left[\mathrm{S}_{0} @ \mathrm{~S}_{0}\right]$ & the population of $\mathrm{S}_{0}$ at the $\mathrm{S}_{0}$ geometry \\
{$\left[\mathrm{S}_{1} @ \mathrm{~S}_{0}\right]$} & the population of $\mathrm{S}_{1}$ at the $\mathrm{S}_{0}$ geometry \\
{$\left[\mathrm{T}_{1} @ \mathrm{~S}_{0}\right]$} & the population of $\mathrm{T}_{1}$ at the $\mathrm{S}_{0}$ geometry \\
{$\left[\mathrm{T}_{2} @ \mathrm{~S}_{0}\right]$} & the population of $\mathrm{T}_{2}$ at the $\mathrm{S}_{0}$ geometry \\
{$\left[\mathrm{T}_{3} @ \mathrm{~S}_{0}\right]$} & the population of $\mathrm{T}_{3}$ at the $\mathrm{S}_{0}$ geometry
\end{tabular}

$\left[\mathrm{S}_{0} @ \mathrm{~S}_{1}\right] \quad$ the population of $\mathrm{S}_{0}$ at the $\mathrm{S}_{1}$ geometry

$\left[\mathrm{S}_{1} @ \mathrm{~S}_{1}\right] \quad$ the population of $\mathrm{S}_{1}$ at the $\mathrm{S}_{1}$ geometry

$\left[\mathrm{T}_{1} @ \mathrm{~S}_{1}\right] \quad$ the population of $\mathrm{T}_{1}$ at the $\mathrm{S}_{1}$ geometry

$\left[\mathrm{T}_{2} @ \mathrm{~S}_{1}\right] \quad$ the population of $\mathrm{T}_{2}$ at the $\mathrm{S}_{1}$ geometry

$\left[\mathrm{T}_{3} @ \mathrm{~S}_{1}\right] \quad$ the population of $\mathrm{T}_{3}$ at the $\mathrm{S}_{1}$ geometry

$\left[\mathrm{S}_{0} @ \mathrm{~T}_{1}\right] \quad$ the population of $\mathrm{S}_{0}$ at the $\mathrm{T}_{1}$ geometry

$\left[\mathrm{S}_{1} @ \mathrm{~T}_{1}\right] \quad$ the population of $\mathrm{S}_{1}$ at the $\mathrm{T}_{1}$ geometry

[ $\left.\mathrm{T}_{1} @ \mathrm{~T}_{1}\right] \quad$ the population of $\mathrm{T}_{1}$ at the $\mathrm{T}_{1}$ geometry

$\left[\mathrm{T}_{2} @ \mathrm{~T}_{1}\right] \quad$ the population of $\mathrm{T}_{2}$ at the $\mathrm{T}_{1}$ geometry

$\left[\mathrm{T}_{3} @ \mathrm{~T}_{1}\right] \quad$ the population of $\mathrm{T}_{3}$ at the $\mathrm{T}_{1}$ geometry

$\left[\mathrm{S}_{0} @ \mathrm{~T}_{3}\right] \quad$ the population of $\mathrm{S}_{0}$ at the $\mathrm{T}_{3}$ geometry

$\left[\mathrm{S}_{1} @ \mathrm{~T}_{3}\right] \quad$ the population of $\mathrm{S}_{1}$ at the $\mathrm{T}_{3}$ geometry

$\left[\mathrm{T}_{1} @ \mathrm{~T}_{3}\right] \quad$ the population of $\mathrm{T}_{1}$ at the $\mathrm{T}_{3}$ geometry

$\left[\mathrm{T}_{2} @ \mathrm{~T}_{3}\right] \quad$ the population of $\mathrm{T}_{2}$ at the $\mathrm{T}_{3}$ geometry

$\left[\mathrm{T}_{3} @ \mathrm{~T}_{3}\right]$ the population of $\mathrm{T}_{3}$ at the $\mathrm{T}_{3}$ geometry

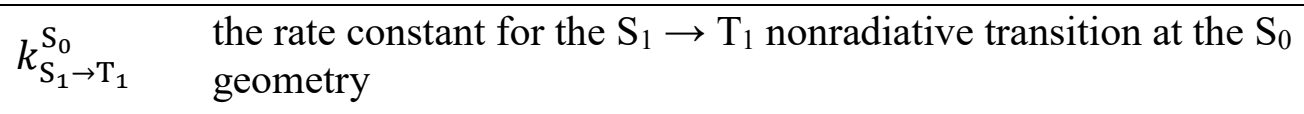

$k_{\mathrm{S}_{1} \rightarrow \mathrm{T}_{2}}^{\mathrm{S}_{0}} \quad$ the rate for the $\mathrm{S}_{1} \rightarrow \mathrm{T}_{2}$ nonradiative transition at the $\mathrm{S}_{0}$ geometry 
$k_{\mathrm{S}_{1} \rightarrow \mathrm{T}_{3}}^{\mathrm{S}_{0}} \quad$ the rate for the $\mathrm{S}_{1} \rightarrow \mathrm{T}_{3}$ nonradiative transition at the $\mathrm{S}_{0}$ geometry

$k_{\mathrm{S}_{1} \rightarrow \mathrm{S}_{0}}^{\mathrm{S}_{0}} \quad$ the rate for the $\mathrm{S}_{1} \rightarrow \mathrm{S}_{0}$ nonradiative decay at the $\mathrm{S}_{0}$ geometry

$k_{\mathrm{F}}^{\mathrm{S}_{0}} \quad$ the rate for the $\mathrm{S}_{1} \rightarrow \mathrm{S}_{0}$ radiative decay at the $\mathrm{S}_{0}$ geometry

$k_{\mathrm{T}_{1} \rightarrow \mathrm{S}_{1}}^{\mathrm{S}_{0}} \quad$ the rate for the $\mathrm{T}_{1} \rightarrow \mathrm{S}_{1}$ nonradiative transition at the $\mathrm{S}_{0}$ geometry

$k_{\mathrm{T}_{2} \rightarrow \mathrm{S}_{1}}^{\mathrm{S}_{0}} \quad$ the rate for the $\mathrm{T}_{2} \rightarrow \mathrm{S}_{1}$ nonradiative transition at the $\mathrm{S}_{0}$ geometry

$k_{\mathrm{T}_{3} \rightarrow \mathrm{S}_{1}}^{\mathrm{S}_{0}} \quad$ the rate for the $\mathrm{T}_{3} \rightarrow \mathrm{S}_{1}$ nonradiative transition at the $\mathrm{S}_{0}$ geometry

$k_{\mathrm{T}_{1} \rightarrow \mathrm{T}_{2}}^{\mathrm{S}_{\mathrm{O}}} \quad$ the rate for the $\mathrm{T}_{1} \rightarrow \mathrm{T}_{2}$ nonradiative transition at the $\mathrm{S}_{0}$ geometry

$k_{\mathrm{T}_{1} \rightarrow \mathrm{T}_{3}}^{\mathrm{S}_{0}} \quad$ the rate for the $\mathrm{T}_{1} \rightarrow \mathrm{T}_{3}$ nonradiative transition at the $\mathrm{S}_{0}$ geometry

$k_{\mathrm{T}_{1} \rightarrow \mathrm{S}_{0}}^{\mathrm{S}_{0}} \quad$ the rate for the $\mathrm{T}_{1} \rightarrow \mathrm{S}_{0}$ nonradiative decay at the $\mathrm{S}_{0}$ geometry

$k_{\mathrm{P}}^{\mathrm{S}_{0}} \quad$ the rate for the $\mathrm{T}_{1} \rightarrow \mathrm{S}_{0}$ radiative decay at the $\mathrm{S}_{0}$ geometry

$k_{\mathrm{T}_{2} \rightarrow \mathrm{T}_{1}}^{\mathrm{S}_{0}} \quad$ the rate for the $\mathrm{T}_{2} \rightarrow \mathrm{T}_{1}$ nonradiative transition at the $\mathrm{S}_{0}$ geometry

$k_{\mathrm{T}_{2} \rightarrow \mathrm{T}_{3}}^{\mathrm{S}_{0}} \quad$ the rate for the $\mathrm{T}_{2} \rightarrow \mathrm{T}_{3}$ nonradiative transition at the $\mathrm{S}_{0}$ geometry

$k_{\mathrm{T}_{3} \rightarrow \mathrm{T}_{1}}^{\mathrm{S}_{0}} \quad$ the rate for the $\mathrm{T}_{3} \rightarrow \mathrm{T}_{1}$ nonradiative transition at the $\mathrm{S}_{0}$ geometry

$k_{\mathrm{T}_{3} \rightarrow \mathrm{T}_{2}}^{\mathrm{S}_{0}} \quad$ the rate for the $\mathrm{T}_{3} \rightarrow \mathrm{T}_{2}$ nonradiative transition at the $\mathrm{S}_{0}$ geometry

$k_{\mathrm{S}_{1} \rightarrow \mathrm{T}_{1}}^{\mathrm{S}_{1}} \quad$ the rate for the $\mathrm{S}_{1} \rightarrow \mathrm{T}_{1}$ nonradiative transition at the $\mathrm{S}_{1}$ geometry

$k_{\mathrm{S}_{1} \rightarrow \mathrm{T}_{2}}^{\mathrm{S}_{1}} \quad$ the rate for the $\mathrm{S}_{1} \rightarrow \mathrm{T}_{2}$ nonradiative transition at the $\mathrm{S}_{1}$ geometry

$k_{\mathrm{S}_{1} \rightarrow \mathrm{T}_{3}}^{\mathrm{S}_{1}} \quad$ the rate for the $\mathrm{S}_{1} \rightarrow \mathrm{T}_{3}$ nonradiative transition at the $\mathrm{S}_{1}$ geometry

$k_{\mathrm{S}_{1} \rightarrow \mathrm{S}_{0}}^{\mathrm{S}_{1}} \quad$ the rate for the $\mathrm{S}_{1} \rightarrow \mathrm{S}_{0}$ nonradiative decay at the $\mathrm{S}_{1}$ geometry

$k_{\mathrm{F}}^{\mathrm{S}_{1}} \quad$ the rate for the $\mathrm{S}_{1} \rightarrow \mathrm{S}_{0}$ radiative decay at the $\mathrm{S}_{1}$ geometry

$k_{\mathrm{T}_{1} \rightarrow \mathrm{S}_{1}}^{\mathrm{S}_{1}} \quad$ the rate for the $\mathrm{T}_{1} \rightarrow \mathrm{S}_{1}$ nonradiative transition at the $\mathrm{S}_{1}$ geometry

$k_{\mathrm{T}_{2} \rightarrow \mathrm{S}_{1}}^{\mathrm{S}_{1}} \quad$ the rate for the $\mathrm{T}_{2} \rightarrow \mathrm{S}_{1}$ nonradiative transition at the $\mathrm{S}_{1}$ geometry

$k_{\mathrm{T}_{3} \rightarrow \mathrm{S}_{1}}^{\mathrm{S}_{1}} \quad$ the rate for the $\mathrm{T}_{3} \rightarrow \mathrm{S}_{1}$ nonradiative transition at the $\mathrm{S}_{1}$ geometry 
$k_{\mathrm{T}_{1} \rightarrow \mathrm{T}_{2}}^{\mathrm{S}_{1}} \quad$ the rate for the $\mathrm{T}_{1} \rightarrow \mathrm{T}_{2}$ nonradiative transition at the $\mathrm{S}_{1}$ geometry $k_{\mathrm{T}_{1} \rightarrow \mathrm{T}_{3}}^{S_{1}} \quad$ the rate for the $\mathrm{T}_{1} \rightarrow \mathrm{T}_{3}$ nonradiative transition at the $\mathrm{S}_{1}$ geometry $k_{\mathrm{T}_{1} \rightarrow \mathrm{S}_{0}}^{\mathrm{S}_{1}} \quad$ the rate for the $\mathrm{T}_{1} \rightarrow \mathrm{S}_{0}$ nonradiative decay at the $\mathrm{S}_{1}$ geometry $k_{\mathrm{P}}^{\mathrm{S}_{1}} \quad$ the rate for the $\mathrm{T}_{1} \rightarrow \mathrm{S}_{0}$ radiative decay at the $\mathrm{S}_{1}$ geometry $k_{\mathrm{T}_{2} \rightarrow \mathrm{T}_{1}}^{\mathrm{S}_{1}} \quad$ the rate for the $\mathrm{T}_{2} \rightarrow \mathrm{T}_{1}$ nonradiative transition at the $\mathrm{S}_{1}$ geometry $k_{\mathrm{T}_{2} \rightarrow \mathrm{T}_{3}}^{\mathrm{S}_{1}} \quad$ the rate for the $\mathrm{T}_{2} \rightarrow \mathrm{T}_{3}$ nonradiative transition at the $\mathrm{S}_{1}$ geometry $k_{\mathrm{T}_{3} \rightarrow \mathrm{T}_{1}}^{S_{1}} \quad$ the rate for the $\mathrm{T}_{3} \rightarrow \mathrm{T}_{1}$ nonradiative transition at the $\mathrm{S}_{1}$ geometry $k_{\mathrm{T}_{3} \rightarrow \mathrm{T}_{2}}^{\mathrm{S}_{1}} \quad$ the rate for the $\mathrm{T}_{3} \rightarrow \mathrm{T}_{2}$ nonradiative transition at the $\mathrm{S}_{1}$ geometry $k_{\mathrm{S}_{1} \rightarrow \mathrm{T}_{1}}^{\mathrm{T}_{1}} \quad$ the rate for the $\mathrm{S}_{1} \rightarrow \mathrm{T}_{1}$ nonradiative transition at the $\mathrm{T}_{1}$ geometry $k_{\mathrm{S}_{1} \rightarrow \mathrm{T}_{2}}^{\mathrm{T}_{1}} \quad$ the rate for the $\mathrm{S}_{1} \rightarrow \mathrm{T}_{2}$ nonradiative transition at the $\mathrm{T}_{1}$ geometry $k_{\mathrm{S}_{1} \rightarrow \mathrm{T}_{3}}^{\mathrm{T}_{1}} \quad$ the rate for the $\mathrm{S}_{1} \rightarrow \mathrm{T}_{3}$ nonradiative transition at the $\mathrm{T}_{1}$ geometry $k_{\mathrm{S}_{1} \rightarrow \mathrm{S}_{0}}^{\mathrm{T}_{1}} \quad$ the rate for the $\mathrm{S}_{1} \rightarrow \mathrm{S}_{0}$ nonradiative decay at the $\mathrm{T}_{1}$ geometry $k_{\mathrm{F}}^{\mathrm{T}_{1}} \quad$ the rate for the $\mathrm{S}_{1} \rightarrow \mathrm{S}_{0}$ radiative decay at the $\mathrm{T}_{1}$ geometry $k_{\mathrm{T}_{1} \rightarrow \mathrm{S}_{1}}^{\mathrm{T}_{1}} \quad$ the rate for the $\mathrm{T}_{1} \rightarrow \mathrm{S}_{1}$ nonradiative transition at the $\mathrm{T}_{1}$ geometry $k_{\mathrm{T}_{2} \rightarrow \mathrm{S}_{1}}^{\mathrm{T}_{1}} \quad$ the rate for the $\mathrm{T}_{2} \rightarrow \mathrm{S}_{1}$ nonradiative transition at the $\mathrm{T}_{1}$ geometry $k_{\mathrm{T}_{3} \rightarrow \mathrm{S}_{1}}^{\mathrm{T}_{1}} \quad$ the rate for the $\mathrm{T}_{3} \rightarrow \mathrm{S}_{1}$ nonradiative transition at the $\mathrm{T}_{1}$ geometry $k_{\mathrm{T}_{1} \rightarrow \mathrm{T}_{2}}^{\mathrm{T}_{1}} \quad$ the rate for the $\mathrm{T}_{1} \rightarrow \mathrm{T}_{2}$ nonradiative transition at the $\mathrm{T}_{1}$ geometry $k_{\mathrm{T}_{1} \rightarrow \mathrm{T}_{3}}^{\mathrm{T}_{1}} \quad$ the rate for the $\mathrm{T}_{1} \rightarrow \mathrm{T}_{3}$ nonradiative transition at the $\mathrm{T}_{1}$ geometry $k_{\mathrm{T}_{1} \rightarrow \mathrm{S}_{0}}^{\mathrm{T}_{1}} \quad$ the rate for the $\mathrm{T}_{1} \rightarrow \mathrm{S}_{0}$ nonradiative decay at the $\mathrm{T}_{1}$ geometry $k_{\mathrm{P}}^{\mathrm{T}_{1}} \quad$ the rate for the $\mathrm{T}_{1} \rightarrow \mathrm{S}_{0}$ radiative decay at the $\mathrm{T}_{1}$ geometry $k_{\mathrm{T}_{2} \rightarrow \mathrm{T}_{1}}^{\mathrm{T}_{1}} \quad$ the rate for the $\mathrm{T}_{2} \rightarrow \mathrm{T}_{1}$ nonradiative transition at the $\mathrm{T}_{1}$ geometry $k_{\mathrm{T}_{2} \rightarrow \mathrm{T}_{3}}^{\mathrm{T}_{1}} \quad$ the rate for the $\mathrm{T}_{2} \rightarrow \mathrm{T}_{3}$ nonradiative transition at the $\mathrm{T}_{1}$ geometry 
$k_{\mathrm{T}_{3} \rightarrow \mathrm{T}_{1}}^{\mathrm{T}_{1}} \quad$ the rate for the $\mathrm{T}_{3} \rightarrow \mathrm{T}_{1}$ nonradiative transition at the $\mathrm{T}_{1}$ geometry $k_{\mathrm{T}_{3} \rightarrow \mathrm{T}_{2}}^{\mathrm{T}_{1}} \quad$ the rate for the $\mathrm{T}_{3} \rightarrow \mathrm{T}_{2}$ nonradiative transition at the $\mathrm{T}_{1}$ geometry

$k_{\mathrm{S}_{1} \rightarrow \mathrm{T}_{1}}^{\mathrm{T}_{3}} \quad$ the rate for the $\mathrm{S}_{1} \rightarrow \mathrm{T}_{1}$ nonradiative transition at the $\mathrm{T}_{3}$ geometry

$k_{\mathrm{S}_{1} \rightarrow \mathrm{T}_{2}}^{\mathrm{T}_{3}} \quad$ the rate for the $\mathrm{S}_{1} \rightarrow \mathrm{T}_{2}$ nonradiative transition at the $\mathrm{T}_{3}$ geometry

$k_{\mathrm{S}_{1} \rightarrow \mathrm{T}_{3}}^{\mathrm{T}_{3}} \quad$ the rate for the $\mathrm{S}_{1} \rightarrow \mathrm{T}_{3}$ nonradiative transition at the $\mathrm{T}_{3}$ geometry

$k_{\mathrm{S}_{1} \rightarrow \mathrm{S}_{0}}^{\mathrm{T}_{3}} \quad$ the rate for the $\mathrm{S}_{1} \rightarrow \mathrm{S}_{0}$ nonradiative decay at the $\mathrm{T}_{3}$ geometry

$k_{\mathrm{F}}^{\mathrm{T}_{3}} \quad$ the rate for the $\mathrm{S}_{1} \rightarrow \mathrm{S}_{0}$ radiative decay at the $\mathrm{T}_{3}$ geometry

$k_{\mathrm{T}_{1} \rightarrow \mathrm{S}_{1}}^{\mathrm{T}_{3}} \quad$ the rate for the $\mathrm{T}_{1} \rightarrow \mathrm{S}_{1}$ nonradiative transition at the $\mathrm{T}_{3}$ geometry

$k_{\mathrm{T}_{2} \rightarrow \mathrm{S}_{1}}^{\mathrm{T}_{3}} \quad$ the rate for the $\mathrm{T}_{2} \rightarrow \mathrm{S}_{1}$ nonradiative transition at the $\mathrm{T}_{3}$ geometry

$k_{\mathrm{T}_{3} \rightarrow \mathrm{S}_{1}}^{\mathrm{T}_{3}} \quad$ the rate for the $\mathrm{T}_{3} \rightarrow \mathrm{S}_{1}$ nonradiative transition at the $\mathrm{T}_{3}$ geometry

$k_{\mathrm{T}_{1} \rightarrow \mathrm{T}_{2}}^{\mathrm{T}_{3}} \quad$ the rate for the $\mathrm{T}_{1} \rightarrow \mathrm{T}_{2}$ nonradiative transition at the $\mathrm{T}_{3}$ geometry

$k_{\mathrm{T}_{1} \rightarrow \mathrm{T}_{3}}^{\mathrm{T}_{3}} \quad$ the rate for the $\mathrm{T}_{1} \rightarrow \mathrm{T}_{3}$ nonradiative transition at the $\mathrm{T}_{3}$ geometry

$k_{\mathrm{T}_{1} \rightarrow \mathrm{S}_{0}}^{\mathrm{T}_{3}} \quad$ the rate for the $\mathrm{T}_{1} \rightarrow \mathrm{S}_{0}$ nonradiative decay at the $\mathrm{T}_{3}$ geometry

$k_{\mathrm{P}}^{\mathrm{T}_{3}} \quad$ the rate for the $\mathrm{T}_{1} \rightarrow \mathrm{S}_{0}$ radiative decay at the $\mathrm{T}_{3}$ geometry

$k_{\mathrm{T}_{2} \rightarrow \mathrm{T}_{1}}^{\mathrm{T}_{3}} \quad$ the rate for the $\mathrm{T}_{2} \rightarrow \mathrm{T}_{1}$ nonradiative transition at the $\mathrm{T}_{3}$ geometry

$k_{\mathrm{T}_{2} \rightarrow \mathrm{T}_{3}}^{\mathrm{T}_{3}} \quad$ the rate for the $\mathrm{T}_{2} \rightarrow \mathrm{T}_{3}$ nonradiative transition at the $\mathrm{T}_{3}$ geometry

$k_{\mathrm{T}_{3} \rightarrow \mathrm{T}_{1}}^{\mathrm{T}_{3}} \quad$ the rate for the $\mathrm{T}_{3} \rightarrow \mathrm{T}_{1}$ nonradiative transition at the $\mathrm{T}_{3}$ geometry

$k_{\mathrm{T}_{3} \rightarrow \mathrm{T}_{2}}^{\mathrm{T}_{3}} \quad$ the rate for the $\mathrm{T}_{3} \rightarrow \mathrm{T}_{2}$ nonradiative transition at the $\mathrm{T}_{3}$ geometry

$k_{\mathrm{S}_{0}, \mathrm{GR}} \quad$ the rate for the geometry relaxation in $\mathrm{S}_{0}$

$k_{\mathrm{S}_{1}, \mathrm{GR}} \quad$ the rate for the geometry relaxation in $\mathrm{S}_{1}$

$k_{\mathrm{T}_{1}, \mathrm{GR}} \quad$ the rate for the geometry relaxation in $\mathrm{T}_{1}$

$k_{\mathrm{T}_{2}, \mathrm{GR}} \quad$ the rate for the geometry relaxation in $\mathrm{T}_{2}$ 
$k_{\mathrm{T}_{3}, \mathrm{GR}} \quad$ the rate for the geometry relaxation in $\mathrm{T}_{3}$ 


\section{Detailed population dynamics for $k_{\mathrm{GR}}=0 \mathrm{~s}^{-1}$}

In this condition, the geometry of $\mathrm{BP}$ is fixed to the $\mathrm{S}_{0}$ geometry and only $\mathrm{S}_{0} @ \mathrm{~S}_{0}, \mathrm{~S}_{1} @ \mathrm{~S}_{0}, \mathrm{~T}_{1} @ \mathrm{~S}_{0}$, $\mathrm{T}_{2} @ \mathrm{~S}_{0}$, and $\mathrm{T}_{3} @ \mathrm{~S}_{0}$ are populated. Figure $\mathrm{S} 4 \mathrm{a}$ shows the calculated populations in the time range of $0-0.5$ ns. At first, $T_{2}$ and $T_{3}$ are populated via the $S_{1} \rightarrow T_{2}$ and $S_{1} \rightarrow T_{3}$ ISCs, because these ISCs are far faster than the $\mathrm{S}_{1} \rightarrow \mathrm{T}_{1}$ ISC and the $\mathrm{S}_{1} \rightarrow \mathrm{S}_{0}$ radiative and nonradiative decays (Table 1). To investigate an influence of the $S_{1} \rightarrow T_{3}$ ISC on the population dynamics, we calculated populations by setting the $\mathrm{S}_{1} \rightarrow \mathrm{T}_{3}$ ISC rate constant $\left(k_{\mathrm{S}_{1} \rightarrow \mathrm{T}_{3}}^{\mathrm{S}_{3}}\right)$ to be $0 \mathrm{~s}^{-1}$ (Figure $\mathrm{S} 4 \mathrm{~b}$ ). We expected that $T_{3}$ would not be populated because the $S_{1} \rightarrow T_{3}$ ISC is forbidden. However, against our expectations, the $T_{3}$ population without the $S_{1} \rightarrow T_{3}$ ISC (Figure $S 4 b$ ) was almost as great as that obtained when the fast $\mathrm{S}_{1} \rightarrow \mathrm{T}_{3}$ ISC was allowed (Figure $\mathrm{S} 4 \mathrm{a}$ ). This result indicates that $\mathrm{T}_{3}$ is generated by the fast $T_{2} \rightarrow T_{3}$ IC after the $S_{1} \rightarrow T_{2}$ ISC. Thus, $T_{3}$ is involved in the decay mechanism irrespective of the presence of the $\mathrm{S}_{1} \rightarrow \mathrm{T}_{3}$ ISC. Experimental observations of $\mathrm{T}_{3}$ would be difficult because of the similar time evolution of the populations and small $\mathrm{T}_{2}-\mathrm{T}_{3}$ energy difference of $67 \mathrm{meV}$. Improved energy resolution (for example, through the use of picosecond transient absorption spectroscopy) would enable direct detection of the simultaneous generation of $\mathrm{T}_{2}$ and $\mathrm{T}_{3}$.

Next, $T_{1}$ is populated via the $T_{2} \rightarrow T_{1}$ IC (Figure $S 4 a$ ). The $T_{2}$ and $T_{3}$ populations take their maximum values of 0.46 and 0.03 , respectively, at $t \approx 0.05 \mathrm{~ns}$ and almost vanish when $t \approx 0.5 \mathrm{~ns}$, whereas the $\mathrm{T}_{1}$ population takes its maximum value of 0.92 at $t \approx 0.3 \mathrm{~ns}$. After $0.05 \mathrm{~ns}$, the $\mathrm{T}_{1} \rightarrow$ $\mathrm{S}_{0}$ ISC also occurs and $\mathrm{S}_{0}$ is increased. Figure $\mathrm{S} 4 \mathrm{c}$ shows calculated populations in the time range of 0-15 ns. After $t=0.5 \mathrm{~ns}$, the $\mathrm{T}_{1} \rightarrow \mathrm{S}_{0}$ ISC occurs mainly and $\mathrm{T}_{1}$ almost completely decays to $\mathrm{S}_{0}$ when $t=15 \mathrm{~ns}$ (at $t=15 \mathrm{~ns}$, the populations of $\mathrm{T}_{1}$ and $\mathrm{S}_{0}$ are 0.01 and 0.99 , respectively). Thus, without any geometry relaxation, the dominant decay paths are the indirect $S_{1} \rightarrow T_{2}\left(\leftrightarrow T_{3}\right) \rightarrow T_{1}$ $\rightarrow \mathrm{S}_{0}$ channels (one channel is without $\mathrm{T}_{3}$ and the other is with $\mathrm{T}_{3}$, both channels are shown in Figure S4d). The so-called direct mechanism via the $\mathrm{S}_{1} \rightarrow \mathrm{T}_{1} \mathrm{ISC}$ (rate of $5.1 \times 10^{8} \mathrm{~s}^{-1}$ ) is suppressed by the faster $\mathrm{S}_{1} \rightarrow \mathrm{T}_{2}$ ISC (rate constant is $2.3 \times 10^{10} \mathrm{~s}^{-1}$ ). 

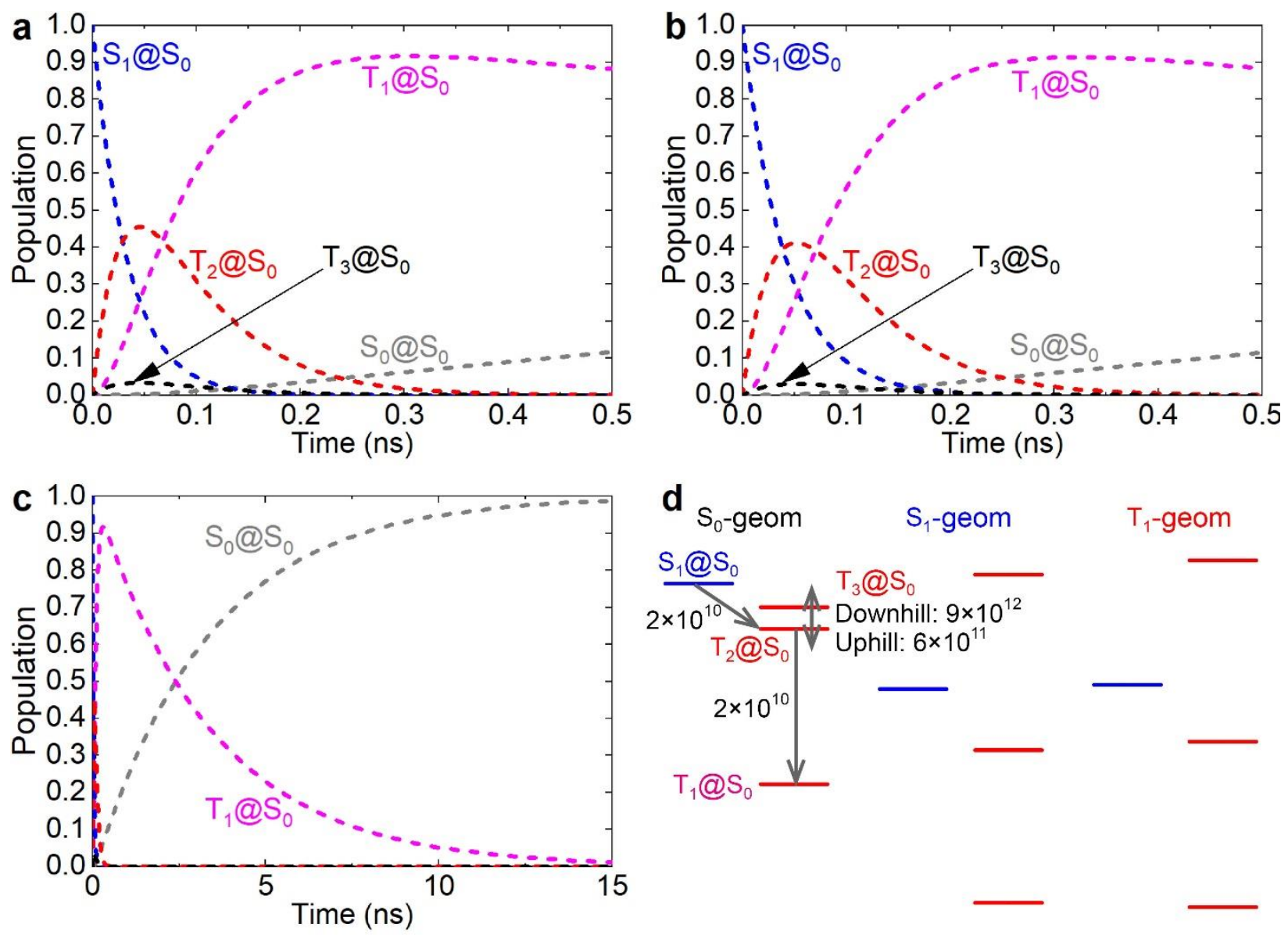

Figure S4. Calculated populations with the rate constants obtained with the simplified formulae when fwhm $=1000 \mathrm{~cm}^{-1}$ and $k_{\mathrm{GR}}=0 \mathrm{~s}^{-1}$ (without any geometry relaxation) in the time range of $(\mathrm{a}, \mathrm{b}) 0-0.5 \mathrm{~ns}$ and (c) $0-15 \mathrm{~ns}$. Figure $\mathrm{S} 4 \mathrm{~b}$ shows the calculated populations by setting the $\mathrm{S}_{1} @ \mathrm{~S}_{0}$ $\rightarrow \mathrm{T}_{3} @ \mathrm{~S}_{0}$ rate constant to be $0 \mathrm{~s}^{-1} . \mathrm{S}_{1} @ \mathrm{~S}_{0}, \mathrm{~T}_{2} @ \mathrm{~S}_{0}$, and $\mathrm{T}_{3} @ \mathrm{~S}_{0}$ in Figure $\mathrm{S} 4 \mathrm{c}$ almost lie on the vertical axis because they are depopulated within $1 \mathrm{~ns}$ and almost zero in the time range of 1-15 ns. (d) The proposed $\mathrm{S}_{1} \rightarrow \mathrm{T}_{2}\left(\leftrightarrow \mathrm{T}_{3}\right) \rightarrow \mathrm{T}_{1} \rightarrow \mathrm{S}_{0}$ decay mechanism for $k_{\mathrm{GR}}=0 \mathrm{~s}^{-1}$.

\section{Detailed population dynamics for $k_{\mathrm{GR}}=1 \times 10^{6} \mathrm{~s}^{-1}$}

Because the $\mathrm{S}_{1} @ \mathrm{~S}_{0} \rightarrow \mathrm{S}_{1} @ \mathrm{~S}_{1}$ geometry relaxation occurs much slower than the $\mathrm{S}_{1} @ \mathrm{~S}_{0} \rightarrow \mathrm{T}_{2} @ \mathrm{~S}_{0}$ ISC $\left(3.0 \times 10^{10} \mathrm{~s}^{-1}\right)$, the molecular geometry is fixed to the $\mathrm{S}_{0}$ geometry during the electronic transitions and the population dynamics are almost identical to those obtained when $k_{\mathrm{GR}}=0 \mathrm{~s}^{-1}$ (Figure S4d). Hence, the decay channel is $\mathrm{S}_{1} \rightarrow \mathrm{T}_{2}\left(\leftrightarrow \mathrm{T}_{3}\right) \rightarrow \mathrm{T}_{1} \rightarrow \mathrm{S}_{0}$ decay mechanism. 


\section{Detailed population dynamics for $k_{\mathrm{GR}}=1 \times 10^{8} \mathrm{~s}^{-1}$}

Because the $\mathrm{S}_{1} @ \mathrm{~S}_{0} \rightarrow \mathrm{T}_{2} @ \mathrm{~S}_{0}$ ISC $\left(2.3 \times 10^{10} \mathrm{~s}^{-1}\right)$ occurs much faster than the $\mathrm{S}_{1} @ \mathrm{~S}_{0} \rightarrow \mathrm{S}_{1} @ \mathrm{~S}_{1}$ geometry relaxation, $\mathrm{T}_{2} @ \mathrm{~S}_{0}$ is dominantly populated and then, $\mathrm{T}_{1} @ \mathrm{~S}_{0}$ is populated via the $\mathrm{T}_{2} @ \mathrm{~S}_{0}$ $\rightarrow \mathrm{T}_{1} @ \mathrm{~S}_{0} \mathrm{IC}$. Because the $\mathrm{T}_{1} @ \mathrm{~S}_{0} \rightarrow \mathrm{S}_{0} @ \mathrm{~S}_{0} \mathrm{ISC}\left(3.0 \times 10^{8} \mathrm{~s}^{-1}\right)$ competes with the $\mathrm{T}_{1} @ \mathrm{~S}_{0} \rightarrow \mathrm{T}_{1} @ \mathrm{~T}_{1}$ geometry relaxation, $\mathrm{S}_{0} @ \mathrm{~S}_{0}$ and $\mathrm{T}_{1} @ \mathrm{~T}_{1}$ are populated simultaneously. $\mathrm{T}_{1} @ \mathrm{~T}_{1}$ is then converted into $\mathrm{S}_{0} @ \mathrm{~T}_{1}$ via the $\mathrm{T}_{1} @ \mathrm{~T}_{1} \rightarrow \mathrm{S}_{0} @ \mathrm{~T}_{1} \mathrm{ISC}$ and finally, $\mathrm{S}_{0} @ \mathrm{~T}_{1}$ is decayed to $\mathrm{S}_{0} @ \mathrm{~S}_{0}$ via the $\mathrm{S}_{0} @ \mathrm{~T}_{1}$ $\rightarrow \mathrm{S}_{0} @ \mathrm{~S}_{0}$ geometry relaxation (Figure $\mathrm{S} 5 \mathrm{a}-\mathrm{c}$ ). Whether BP takes the $\mathrm{T}_{1}$ geometry or not, the dominant decay paths are the indirect $\mathrm{S}_{1} \rightarrow \mathrm{T}_{2}\left(\leftrightarrow \mathrm{T}_{3}\right) \rightarrow \mathrm{T}_{1} \rightarrow \mathrm{S}_{0}$ channels (one channel is without $\mathrm{T}_{3}$ and the other is with $\mathrm{T}_{3}$, both channels are shown in Figure $\mathrm{S} 5 \mathrm{~d}$ )
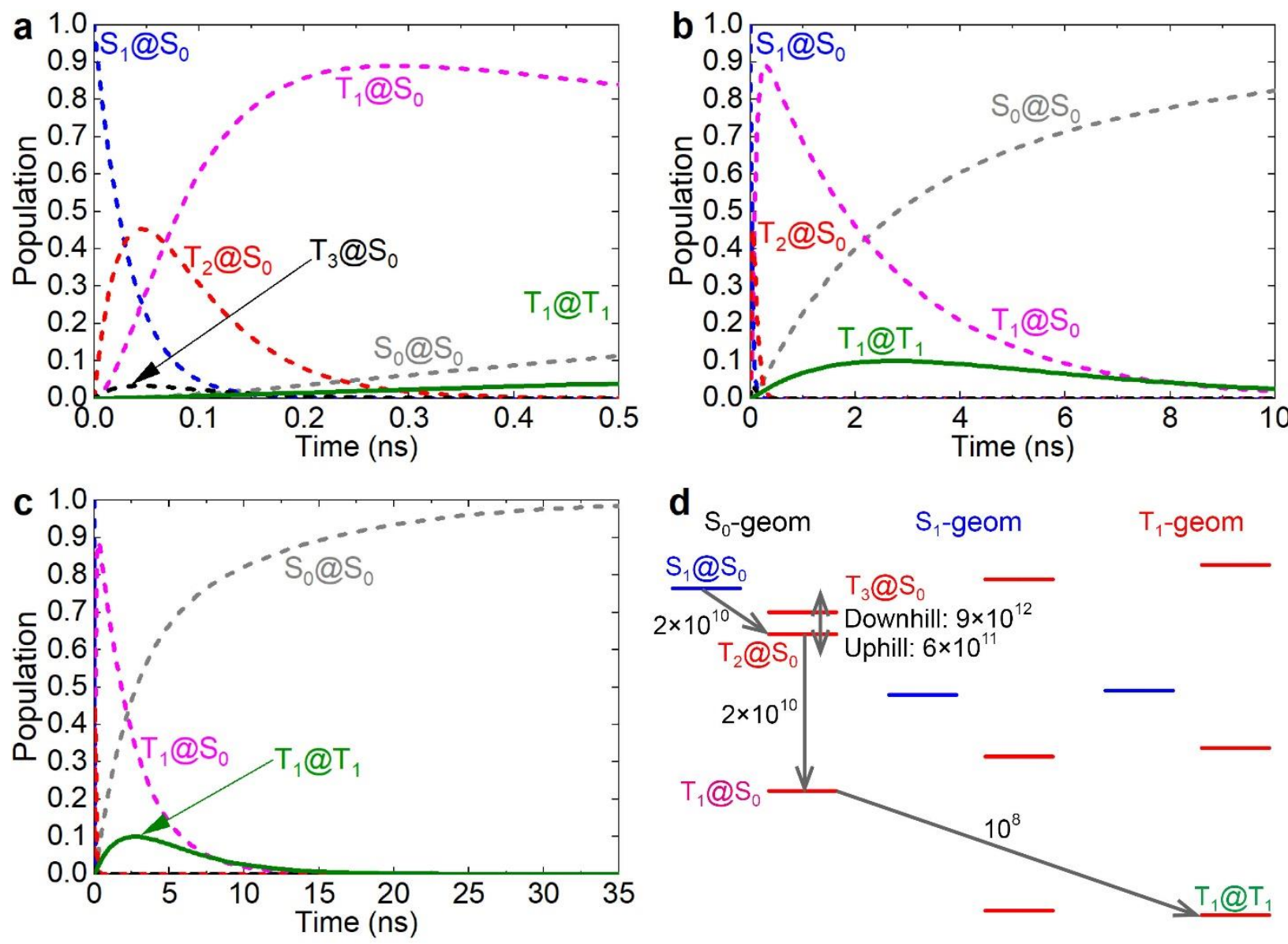

Figure S5. Calculated populations with the rate constants obtained with the simplified formulae when fwhm $=1000 \mathrm{~cm}^{-1}$ and $k_{\mathrm{GR}}=1 \times 10^{8} \mathrm{~s}^{-1}$ in the time range of (a) 0-0.5 ns, (b) 0-10 ns, and (c) 0-15 ns. (d) The proposed $\mathrm{S}_{1} \rightarrow \mathrm{T}_{2}\left(\leftrightarrow \mathrm{T}_{3}\right) \rightarrow \mathrm{T}_{1} \rightarrow \mathrm{S}_{0}$ decay mechanism for $k_{\mathrm{GR}}=1.0 \times 10^{8}$ $\mathrm{s}^{-1}$. 


\section{Detailed population dynamics for $k_{\mathrm{GR}}=1 \times 10^{10} \mathrm{~s}^{-1}$}

Figure S6a shows the calculated populations in the time range of $0-0.35 \mathrm{~ns}$ when $k_{\mathrm{GR}}=1 \times 10^{10} \mathrm{~s}^{-1}$. Because various transitions compete with the geometry relaxations, the time evolution of the populations is rather complicated. For example, $\mathrm{S}_{1} @ \mathrm{~S}_{0}, \mathrm{~S}_{1} @ \mathrm{~S}_{1}, \mathrm{~T}_{2} @ \mathrm{~S}_{0}, \mathrm{~T}_{3} @ \mathrm{~S}_{0}, \mathrm{~T}_{2} @ \mathrm{~S}_{1}, \mathrm{~T}_{1} @ \mathrm{~S}_{0}$, $\mathrm{T}_{1} @ \mathrm{~S}_{1}, \mathrm{~T}_{1} @ \mathrm{~T}_{1}, \mathrm{~S}_{0} @ \mathrm{~S}_{0}$, and $\mathrm{S}_{0} @ \mathrm{~T}_{1}$ coexist. Because the $\mathrm{S}_{1} @ \mathrm{~S}_{0} \rightarrow \mathrm{S}_{1} @ \mathrm{~S}_{1}$ geometry relaxation and $\mathrm{S}_{1} @ \mathrm{~S}_{0} \rightarrow \mathrm{T}_{2} @ \mathrm{~S}_{0} \mathrm{ISC}\left(2.3 \times 10^{10} \mathrm{~s}^{-1}\right)$ occur in the same time scale, $\mathrm{S}_{1} @ \mathrm{~S}_{1}$ and $\mathrm{T}_{2} @ \mathrm{~S}_{0}$ are first increased simultaneously. The generated $\mathrm{S}_{1} @ \mathrm{~S}_{1}$ is immediately converted into $\mathrm{T}_{2} @ \mathrm{~S}_{1}$ via the fast $\mathrm{S}_{1} \rightarrow \mathrm{T}_{2}$ ISC $\left(5.7 \times 10^{10} \mathrm{~s}^{-1}\right)$ and consequently, $\mathrm{T}_{2} @ \mathrm{~S}_{1}$ is populated. $\mathrm{T}_{2} @ \mathrm{~S}_{0}$ and $\mathrm{T}_{2} @ \mathrm{~S}_{1}$ are converted into $\mathrm{T}_{1}$ via the $\mathrm{T}_{2} \rightarrow \mathrm{T}_{1} \mathrm{IC}\left(\sim 10^{11} \mathrm{~s}^{-1}\right)$ and $\mathrm{T}_{1} @ \mathrm{~S}_{0}$ and $\mathrm{T}_{1} @ \mathrm{~S}_{1}$ coexist at the same time. Then, $\mathrm{T}_{1} @ \mathrm{~S}_{0}$ and $\mathrm{T}_{1} @ \mathrm{~S}_{1}$ are converted into $\mathrm{T}_{1} @ \mathrm{~T}_{1}$ via the $\mathrm{T}_{1} @ \mathrm{~S}_{0} \rightarrow \mathrm{T}_{1} @ \mathrm{~T}_{1}$ and $\mathrm{T}_{1} @ \mathrm{~S}_{1} \rightarrow \mathrm{T}_{1} @ \mathrm{~T}_{1}$ geometry relaxations, respectively. The $\mathrm{T}_{1} @ \mathrm{~T}_{1}$ population is increased rapidly and takes its maximum value of 0.87 at $t=0.4$ ns (Figure $\mathrm{S} 6 \mathrm{~b}$ ). After that, $\mathrm{T}_{1} @ \mathrm{~T}_{1}$ is converted into $\mathrm{S}_{0} @ \mathrm{~S}_{0}$ via the $\mathrm{T}_{1} @ \mathrm{~T}_{1} \rightarrow \mathrm{S}_{0} @ \mathrm{~T}_{1} \mathrm{ISC}$ and $\mathrm{S}_{0} @ \mathrm{~T}_{1} \rightarrow \mathrm{S}_{0} @ \mathrm{~S}_{0}$ geometry relaxation. At $t=15 \mathrm{~ns}$, the $\mathrm{S}_{0} @ \mathrm{~S}_{0}$ population is nearly 1, suggesting that all the excited states are completely decayed to the ground state. Thus, when $k_{\mathrm{GR}}=1.0 \times 10^{10} \mathrm{~s}^{-1}$, the dominant triplet population paths are the indirect $\mathrm{S}_{1} \rightarrow$ $\mathrm{T}_{2}\left(\leftrightarrow \mathrm{T}_{3}\right) \rightarrow \mathrm{T}_{1} \rightarrow \mathrm{S}_{0}$ channels (one channel is without $\mathrm{T}_{3}$ and the other is with $\mathrm{T}_{3}$, both channels are shown in Figure S6d) 

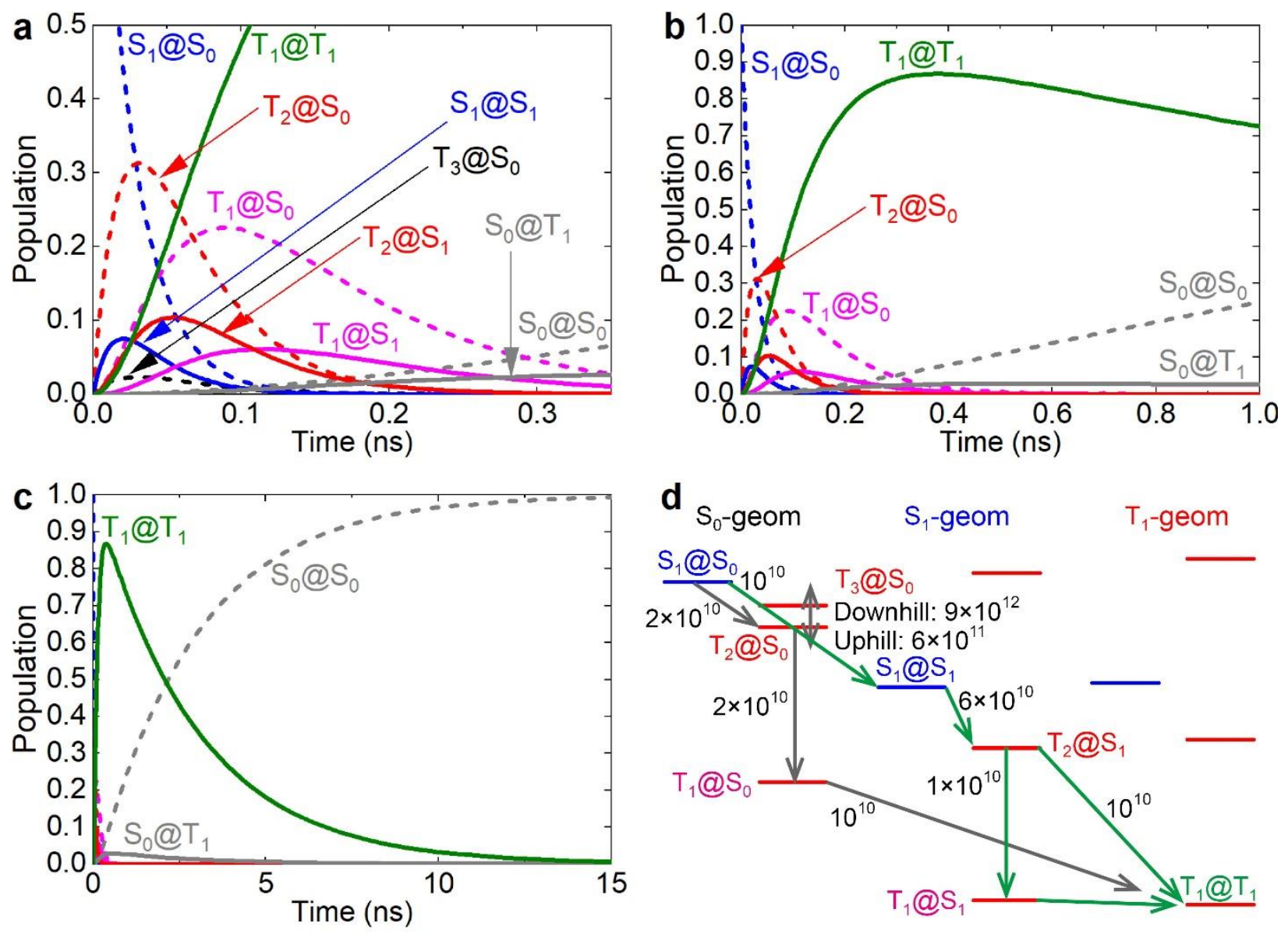

Figure S6. Calculated populations with the rate constants obtained with the simplified formulae when fwhm $=1000 \mathrm{~cm}^{-1}$ and $k_{\mathrm{GR}}=1 \times 10^{10} \mathrm{~s}^{-1}$ in the time range of (a) $0-0.35 \mathrm{~ns}$, (b) $0-1 \mathrm{~ns}$, and (c) 0-15 ns. (d) The proposed $\mathrm{S}_{1} \rightarrow \mathrm{T}_{2}\left(\leftrightarrow \mathrm{T}_{3}\right) \rightarrow \mathrm{T}_{1} \rightarrow \mathrm{S}_{0}$ decay mechanism for $k_{\mathrm{GR}}=1.0 \times 10^{10}$ $\mathrm{s}^{-1}$. 
Table S14. Nonradiative and radiative transition rate constants $\left(\mathrm{s}^{-1}\right)$ calculated for the $\mathrm{S}_{0}, \mathrm{~S}_{1}, \mathrm{~T}_{1}$, and $\mathrm{T}_{3}$ geometries of BP. The fwhm of the Lorentzian broadening was set to be $600 \mathrm{~cm}^{-1}$.

\begin{tabular}{|c|c|c|c|c|}
\hline & $\mathrm{S}_{0}$ geom & $\mathrm{S}_{1}$ geom & $\mathrm{T}_{1}$ geom & $\mathrm{T}_{3}$ geom \\
\hline \multicolumn{5}{|c|}{ Nonradiative transition (ISC) } \\
\hline $\mathrm{S}_{1} \rightarrow \mathrm{T}_{1}$ & $3.1 \times 10^{8 *}$ & $8.0 \times 10^{8}$ & $8.6 \times 10^{8}$ & $8.2 \times 10^{8}$ \\
\hline $\mathrm{S}_{1} \rightarrow \mathrm{T}_{2}$ & $1.5 \times 10^{10}$ & $3.6 \times 10^{10}$ & $3.7 \times 10^{10}$ & $1.6 \times 10^{10}$ \\
\hline $\mathrm{S}_{1} \rightarrow \mathrm{T}_{3}$ & $5.6 \times 10^{9}$ & $1.7 \times 10^{4}$ & $7.2 \times 10^{3}$ & $4.8 \times 10^{9}$ \\
\hline $\mathrm{T}_{1} \rightarrow \mathrm{S}_{0}$ & $1.8 \times 10^{8}$ & $2.0 \times 10^{8}$ & $2.1 \times 10^{8}$ & $1.1 \times 10^{8}$ \\
\hline $\mathrm{T}_{1} \rightarrow \mathrm{S}_{1}$ & $3.0 \times 10^{-3}$ & $1.7 \times 10^{-3}$ & $6.0 \times 10^{-4}$ & $1.5 \times 10^{-3}$ \\
\hline $\mathrm{T}_{2} \rightarrow \mathrm{S}_{0}$ & $3.9 \times 10^{7}$ & $5.6 \times 10^{7}$ & $6.2 \times 10^{7}$ & $7.5 \times 10^{7}$ \\
\hline $\mathrm{T}_{2} \rightarrow \mathrm{S}_{1}$ & $2.1 \times 10^{7}$ & $7.6 \times 10^{6}$ & $1.2 \times 10^{7}$ & $1.3 \times 10^{5}$ \\
\hline $\mathrm{T}_{3} \rightarrow \mathrm{S}_{0}$ & $1.1 \times 10^{5}$ & $4.4 \times 10^{5}$ & $6.4 \times 10^{5}$ & $5.4 \times 10^{4}$ \\
\hline $\mathrm{T}_{3} \rightarrow \mathrm{S}_{1}$ & $1.1 \times 10^{8}$ & $5.7 \times 10^{9}$ & $7.9 \times 10^{9}$ & $2.0 \times 10^{5}$ \\
\hline \multicolumn{5}{|c|}{ Nonradiative transition (IC) } \\
\hline $\mathrm{S}_{1} \rightarrow \mathrm{S}_{0}$ & $\begin{array}{l}2.3 \times 10^{6 *} \\
1.1 \times 10^{8 * *}\end{array}$ & $\begin{array}{l}2.0 \times 10^{6} \\
1.1 \times 10^{8}\end{array}$ & $\begin{array}{l}2.0 \times 10^{6} \\
1.1 \times 10^{8}\end{array}$ & $\begin{array}{l}2.2 \times 10^{6} \\
1.8 \times 10^{8}\end{array}$ \\
\hline $\mathrm{T}_{1} \rightarrow \mathrm{T}_{2}$ & $\begin{array}{l}7.2 \times 10^{1} \\
1.1 \times 10^{3}\end{array}$ & $\begin{array}{l}7.0 \times 10^{1} \\
2.3 \times 10^{3}\end{array}$ & $\begin{array}{l}1.2 \times 10^{1} \\
2.8 \times 10^{1}\end{array}$ & $\begin{array}{l}4.7 \times 10^{3} \\
4.0 \times 10^{5}\end{array}$ \\
\hline $\mathrm{T}_{1} \rightarrow \mathrm{T}_{3}$ & $\begin{array}{c}8.3 \times 10^{-1} \\
1.6 \times 10^{1}\end{array}$ & $\begin{array}{l}3.0 \times 10^{-9} \\
3.4 \times 10^{-8}\end{array}$ & $\begin{array}{c}2.7 \times 10^{-10} \\
1.0 \times 10^{-9}\end{array}$ & $\begin{array}{l}6.5 \times 10^{2} \\
5.5 \times 10^{3}\end{array}$ \\
\hline $\mathrm{T}_{2} \rightarrow \mathrm{T}_{1}$ & $\begin{array}{l}1.1 \times 10^{10} \\
1.6 \times 10^{11}\end{array}$ & $\begin{array}{c}7.1 \times 10^{9} \\
2.3 \times 10^{11}\end{array}$ & $\begin{array}{l}5.9 \times 10^{9} \\
1.3 \times 10^{10}\end{array}$ & $\begin{array}{l}2.0 \times 10^{10} \\
1.7 \times 10^{10}\end{array}$ \\
\hline $\mathrm{T}_{2} \rightarrow \mathrm{T}_{3}$ & $\begin{array}{l}5.6 \times 10^{11} \\
3.1 \times 10^{13}\end{array}$ & $\begin{array}{l}5.1 \times 10^{1} \\
3.6 \times 10^{3}\end{array}$ & $\begin{array}{l}1.7 \times 10^{1} \\
1.5 \times 10^{3}\end{array}$ & $\begin{array}{l}8.7 \times 10^{11} \\
2.2 \times 10^{14}\end{array}$ \\
\hline $\mathrm{T}_{3} \rightarrow \mathrm{T}_{1}$ & $\begin{array}{c}1.5 \times 10^{9} \\
3.1 \times 10^{10}\end{array}$ & $\begin{array}{l}4.7 \times 10^{8} \\
5.5 \times 10^{9}\end{array}$ & $\begin{array}{l}4.2 \times 10^{8} \\
1.6 \times 10^{9}\end{array}$ & $\begin{array}{l}1.4 \times 10^{10} \\
1.2 \times 10^{11}\end{array}$ \\
\hline $\mathrm{T}_{3} \rightarrow \mathrm{T}_{2}$ & $\begin{array}{l}7.5 \times 10^{12} \\
4.2 \times 10^{14}\end{array}$ & $\begin{array}{l}8.1 \times 10^{10} \\
5.8 \times 10^{12}\end{array}$ & $\begin{array}{l}5.5 \times 10^{10} \\
4.8 \times 10^{12}\end{array}$ & $\begin{array}{l}4.4 \times 10^{12} \\
1.1 \times 10^{15}\end{array}$ \\
\hline
\end{tabular}

*: The IC and ISC rate constants calculated using the simplified method.

**: The IC rate constants calculated using the Fourier transformed method. 

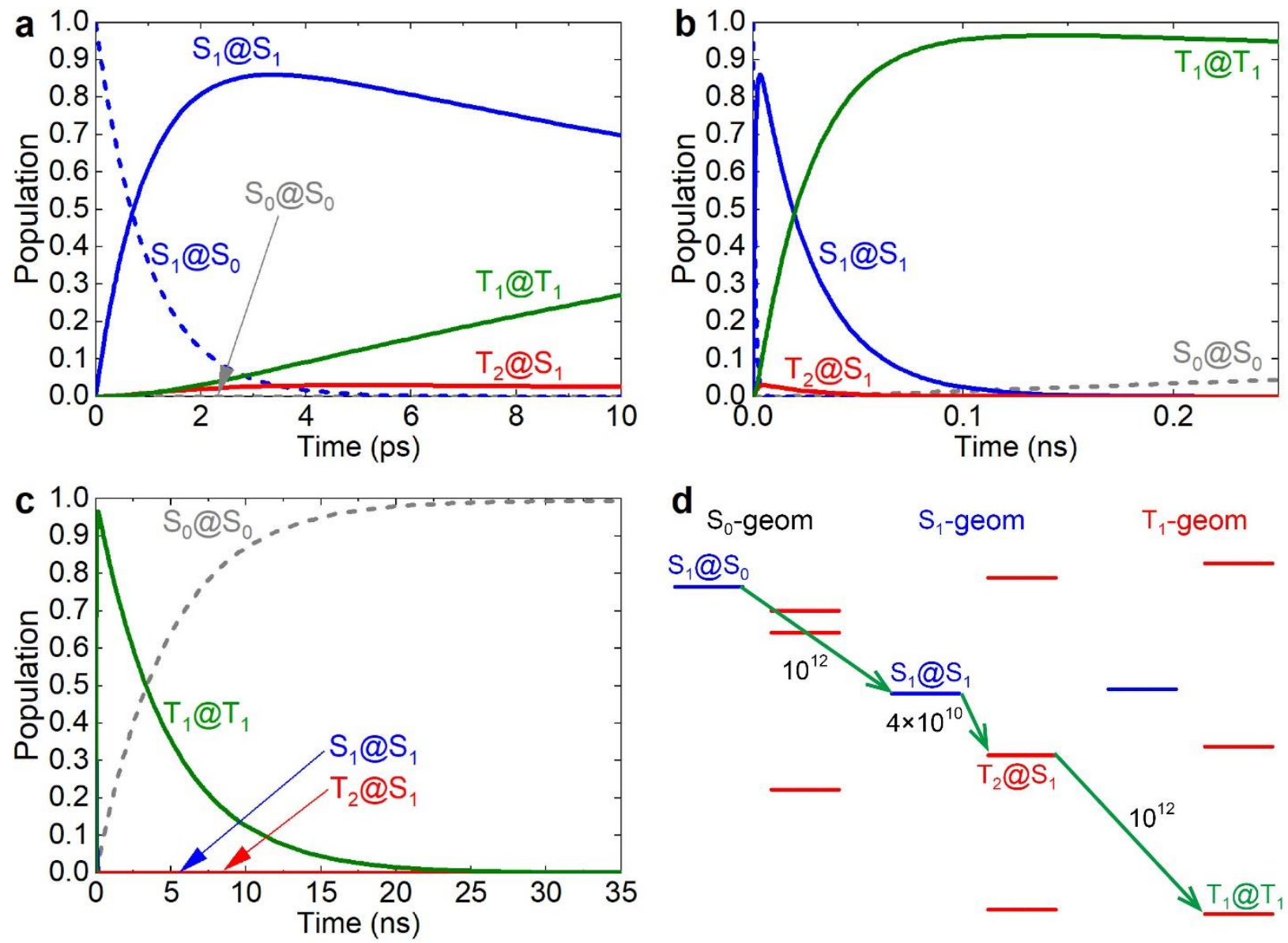

Figure S7. Calculated populations with the rate constants obtained with the simplified formulae when fwhm $=600 \mathrm{~cm}^{-1}$ and $k_{\mathrm{GR}}=1 \times 10^{12} \mathrm{~s}^{-1}$ in the time range of (a) $0-10 \mathrm{ps}$, (b) $0-0.25 \mathrm{~ns}$, and (c) 0-35 ns. (d) The proposed $\mathrm{T}_{2} @ \mathrm{~S}_{1}$ mediated $\mathrm{S}_{1} @ \mathrm{~S}_{0} \rightarrow \mathrm{S}_{1} @ \mathrm{~S}_{1} \rightarrow \mathrm{T}_{2} @ \mathrm{~S}_{1} \rightarrow \mathrm{T}_{1} @ \mathrm{~T}_{1} \rightarrow \mathrm{S}_{0} @ \mathrm{~S}_{0}$ decay mechanism. 
Table S15. Nonradiative and radiative transition rate constants $\left(\mathrm{s}^{-1}\right)$ calculated for the $\mathrm{S}_{0}, \mathrm{~S}_{1}, \mathrm{~T}_{1}$, and $\mathrm{T}_{3}$ geometries of BP. The fwhm of the Lorentzian broadening was set to be $1400 \mathrm{~cm}^{-1}$.

\begin{tabular}{|c|c|c|c|c|}
\hline & $\mathrm{S}_{0}$ geom & $\mathrm{S}_{1}$ geom & $\mathrm{T}_{1}$ geom & $\mathrm{T}_{3}$ geom \\
\hline \multicolumn{5}{|c|}{ Nonradiative transition (ISC) } \\
\hline $\mathrm{S}_{1} \rightarrow \mathrm{T}_{1}$ & $7.1 \times 10^{8 *}$ & $1.8 \times 10^{9}$ & $2.0 \times 10^{9}$ & $1.9 \times 10^{9}$ \\
\hline $\mathrm{S}_{1} \rightarrow \mathrm{T}_{2}$ & $3.0 \times 10^{10}$ & $7.3 \times 10^{10}$ & $7.3 \times 10^{10}$ & $3.2 \times 10^{10}$ \\
\hline $\mathrm{S}_{1} \rightarrow \mathrm{T}_{3}$ & $5.8 \times 10^{9}$ & $1.7 \times 10^{4}$ & $7.4 \times 10^{3}$ & $5.0 \times 10^{9}$ \\
\hline $\mathrm{T}_{1} \rightarrow \mathrm{S}_{0}$ & $4.2 \times 10^{8}$ & $4.7 \times 10^{8}$ & $4.8 \times 10^{8}$ & $2.7 \times 10^{8}$ \\
\hline $\mathrm{T}_{1} \rightarrow \mathrm{S}_{1}$ & $7.1 \times 10^{-3}$ & $3.8 \times 10^{-3}$ & $1.4 \times 10^{-3}$ & $3.5 \times 10^{-3}$ \\
\hline $\mathrm{T}_{2} \rightarrow \mathrm{S}_{0}$ & $9.1 \times 10^{7}$ & $1.3 \times 10^{8}$ & $1.4 \times 10^{8}$ & $1.8 \times 10^{8}$ \\
\hline $\mathrm{T}_{2} \rightarrow \mathrm{S}_{1}$ & $4.2 \times 10^{7}$ & $1.5 \times 10^{7}$ & $2.5 \times 10^{7}$ & $2.5 \times 10^{5}$ \\
\hline $\mathrm{T}_{3} \rightarrow \mathrm{S}_{0}$ & $2.6 \times 10^{5}$ & $1.0 \times 10^{6}$ & $1.5 \times 10^{6}$ & $1.3 \times 10^{5}$ \\
\hline $\mathrm{T}_{3} \rightarrow \mathrm{S}_{1}$ & $1.1 \times 10^{8}$ & $5.9 \times 10^{9}$ & $8.2 \times 10^{9}$ & $2.0 \times 10^{5}$ \\
\hline \multicolumn{5}{|c|}{ Nonradiative transition (IC) } \\
\hline $\mathrm{S}_{1} \rightarrow \mathrm{S}_{0}$ & $\begin{array}{l}5.3 \times 10^{6 *} \\
1.1 \times 10^{8 * *}\end{array}$ & $\begin{array}{l}4.6 \times 10^{6} \\
1.1 \times 10^{8}\end{array}$ & $\begin{array}{l}4.7 \times 10^{6} \\
1.1 \times 10^{8}\end{array}$ & $\begin{array}{l}5.2 \times 10^{6} \\
1.8 \times 10^{8}\end{array}$ \\
\hline $\mathrm{T}_{1} \rightarrow \mathrm{T}_{2}$ & $\begin{array}{l}1.6 \times 10^{2} \\
1.1 \times 10^{3}\end{array}$ & $\begin{array}{l}1.5 \times 10^{2} \\
2.3 \times 10^{3}\end{array}$ & $\begin{array}{l}1.5 \times 10^{1} \\
2.8 \times 10^{1}\end{array}$ & $\begin{array}{l}1.0 \times 10^{4} \\
4.0 \times 10^{5}\end{array}$ \\
\hline $\mathrm{T}_{1} \rightarrow \mathrm{T}_{3}$ & $\begin{array}{c}1.9 \\
1.6 \times 10^{1}\end{array}$ & $\begin{array}{l}6.8 \times 10^{-9} \\
3.4 \times 10^{-8}\end{array}$ & $\begin{array}{c}6.2 \times 10^{-10} \\
1.0 \times 10^{-9}\end{array}$ & $\begin{array}{l}1.4 \times 10^{3} \\
5.5 \times 10^{3}\end{array}$ \\
\hline $\mathrm{T}_{2} \rightarrow \mathrm{T}_{1}$ & $\begin{array}{l}2.2 \times 10^{10} \\
1.6 \times 10^{11}\end{array}$ & $\begin{array}{l}1.5 \times 10^{10} \\
2.3 \times 10^{11}\end{array}$ & $\begin{array}{l}1.3 \times 10^{10} \\
1.3 \times 10^{10}\end{array}$ & $\begin{array}{l}4.4 \times 10^{10} \\
1.7 \times 10^{10}\end{array}$ \\
\hline $\mathrm{T}_{2} \rightarrow \mathrm{T}_{3}$ & $\begin{array}{l}6.4 \times 10^{11} \\
3.1 \times 10^{13}\end{array}$ & $\begin{array}{l}6.8 \times 10^{1} \\
3.6 \times 10^{3}\end{array}$ & $\begin{array}{l}2.4 \times 10^{1} \\
1.5 \times 10^{3}\end{array}$ & $\begin{array}{l}1.6 \times 10^{12} \\
2.2 \times 10^{14}\end{array}$ \\
\hline $\mathrm{T}_{3} \rightarrow \mathrm{T}_{1}$ & $\begin{array}{l}3.6 \times 10^{9} \\
3.1 \times 10^{10}\end{array}$ & $\begin{array}{l}1.1 \times 10^{9} \\
5.5 \times 10^{9}\end{array}$ & $\begin{array}{l}9.6 \times 10^{8} \\
1.6 \times 10^{9}\end{array}$ & $\begin{array}{l}3.1 \times 10^{10} \\
1.2 \times 10^{11}\end{array}$ \\
\hline $\mathrm{T}_{3} \rightarrow \mathrm{T}_{2}$ & $\begin{array}{l}8.6 \times 10^{12} \\
4.2 \times 10^{14}\end{array}$ & $\begin{array}{l}1.1 \times 10^{11} \\
5.8 \times 10^{12}\end{array}$ & $\begin{array}{l}8.0 \times 10^{10} \\
4.8 \times 10^{12}\end{array}$ & $\begin{array}{l}8.2 \times 10^{12} \\
1.1 \times 10^{15}\end{array}$ \\
\hline
\end{tabular}

*: The IC and ISC rate constants calculated using the simplified method.

**: The IC rate constants calculated using the Fourier transformed method. 

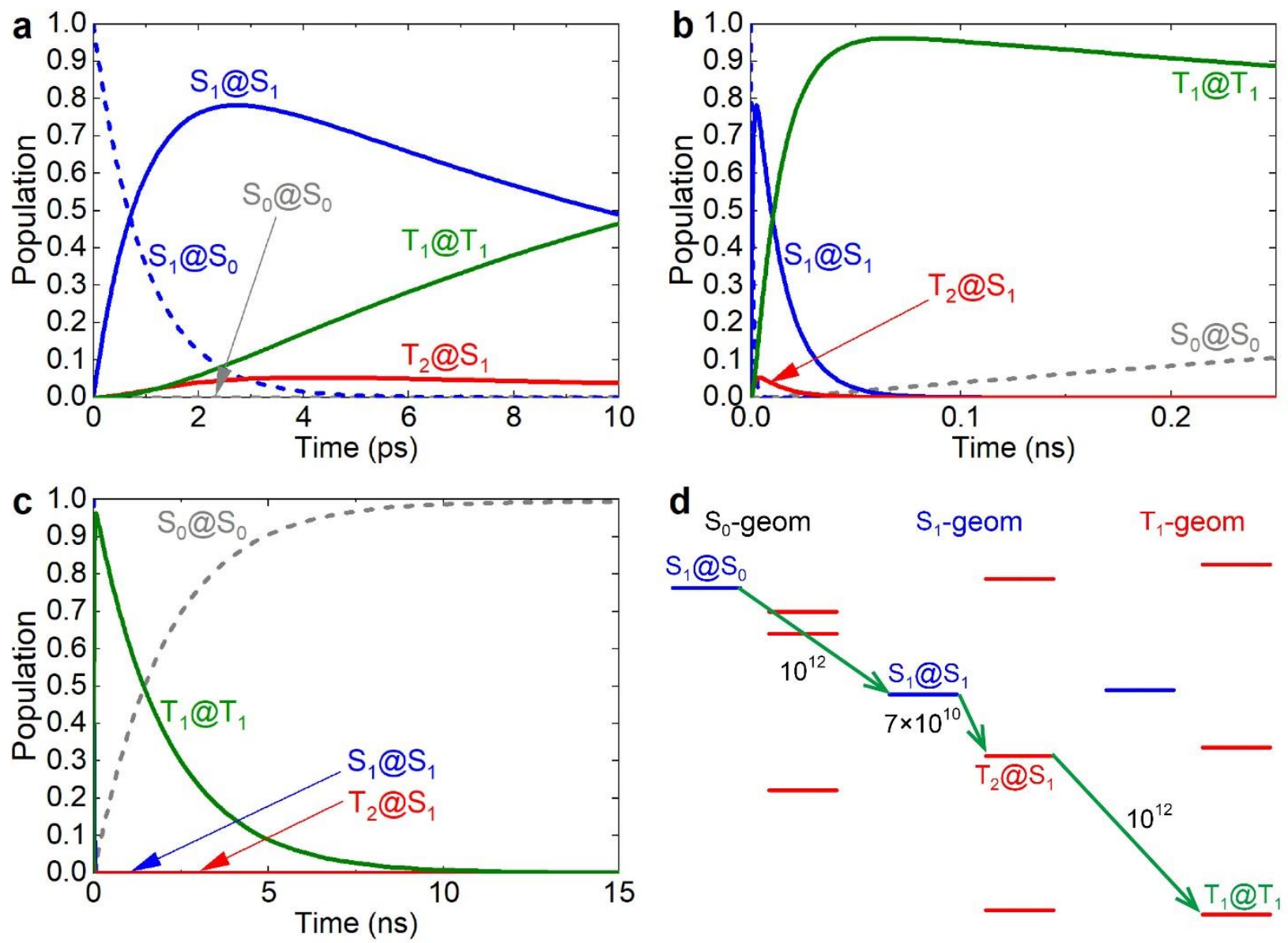

Figure S8. Calculated populations with the rate constants obtained with the simplified formulae when fwhm $=1400 \mathrm{~cm}^{-1}$ and $k_{\mathrm{GR}}=1 \times 10^{12} \mathrm{~s}^{-1}$ in the time range of (a) 0-10 ps, (b) 0-0.25 ns, and (c) 0-15 ns. (d) The proposed $\mathrm{T}_{2} @ \mathrm{~S}_{1}$ mediated $\mathrm{S}_{1} @ \mathrm{~S}_{0} \rightarrow \mathrm{S}_{1} @ \mathrm{~S}_{1} \rightarrow \mathrm{T}_{2} @ \mathrm{~S}_{1} \rightarrow \mathrm{T}_{1} @ \mathrm{~T}_{1} \rightarrow \mathrm{S}_{0} @ \mathrm{~S}_{0}$ decay mechanism. 

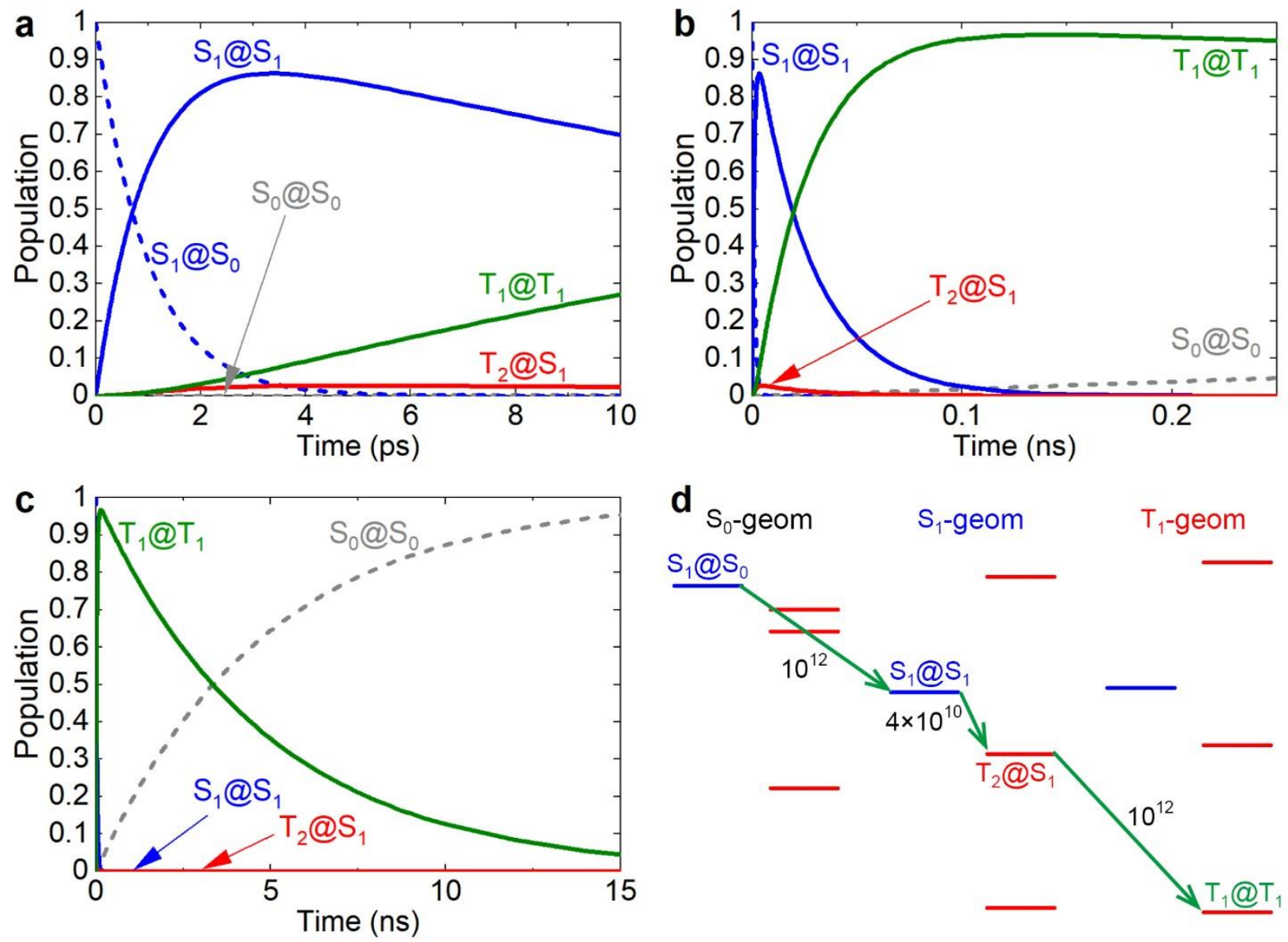

Figure S9. Calculated populations with the rate constants obtained with the Fourier transformed formulae when fwhm $=600 \mathrm{~cm}^{-1}$ and $k_{\mathrm{GR}}=1 \times 10^{12} \mathrm{~s}^{-1}$ in the time range of (a) $0-10 \mathrm{ps}$, (b) 0 $0.25 \mathrm{~ns}$, and (c) 0-15 ns. (d) The proposed $\mathrm{T}_{2} @ \mathrm{~S}_{1}$ mediated $\mathrm{S}_{1} @ \mathrm{~S}_{0} \rightarrow \mathrm{S}_{1} @ \mathrm{~S}_{1} \rightarrow \mathrm{T}_{2} @ \mathrm{~S}_{1} \rightarrow \mathrm{T}_{1} @ \mathrm{~T}_{1}$ $\rightarrow \mathrm{S}_{0} @ \mathrm{~S}_{0}$ decay mechanism. 

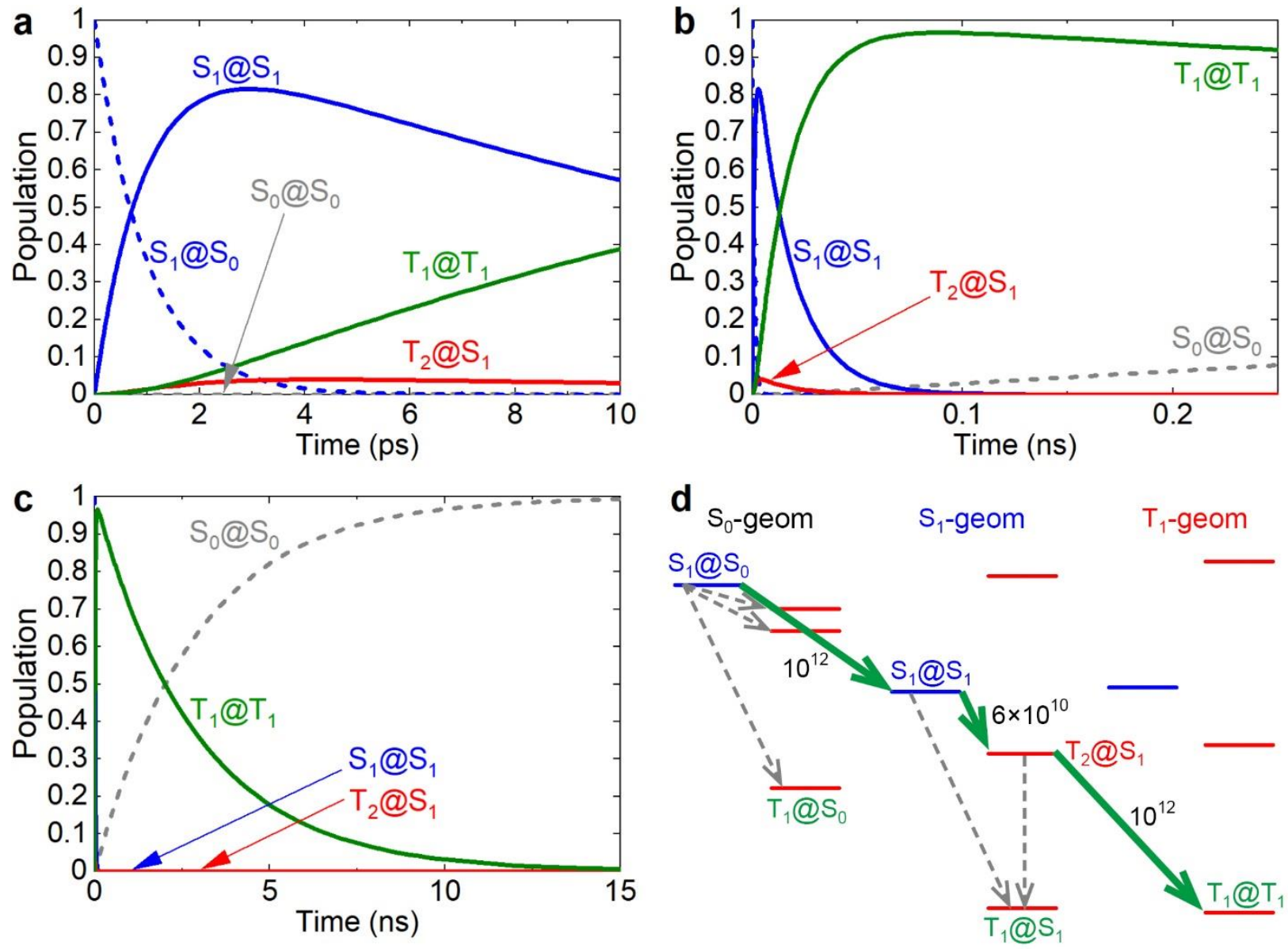

Figure S10. Calculated populations with the rate constants obtained with the Fourier transformed formulae when fwhm $=1000 \mathrm{~cm}^{-1}$ and $k_{\mathrm{GR}}=1 \times 10^{12} \mathrm{~s}^{-1}$ in the time range of (a) 0 10 ps, (b) 0-0.25 ns, and (c) 0-15 ns. (d) The proposed $\mathrm{T}_{2} @ \mathrm{~S}_{1}$ mediated $\mathrm{S}_{1} @ \mathrm{~S}_{0} \rightarrow \mathrm{S}_{1} @ \mathrm{~S}_{1} \rightarrow$ $\mathrm{T}_{2} @ \mathrm{~S}_{1} \rightarrow \mathrm{T}_{1} @ \mathrm{~T}_{1} \rightarrow \mathrm{S}_{0} @ \mathrm{~S}_{0}$ decay mechanism. 

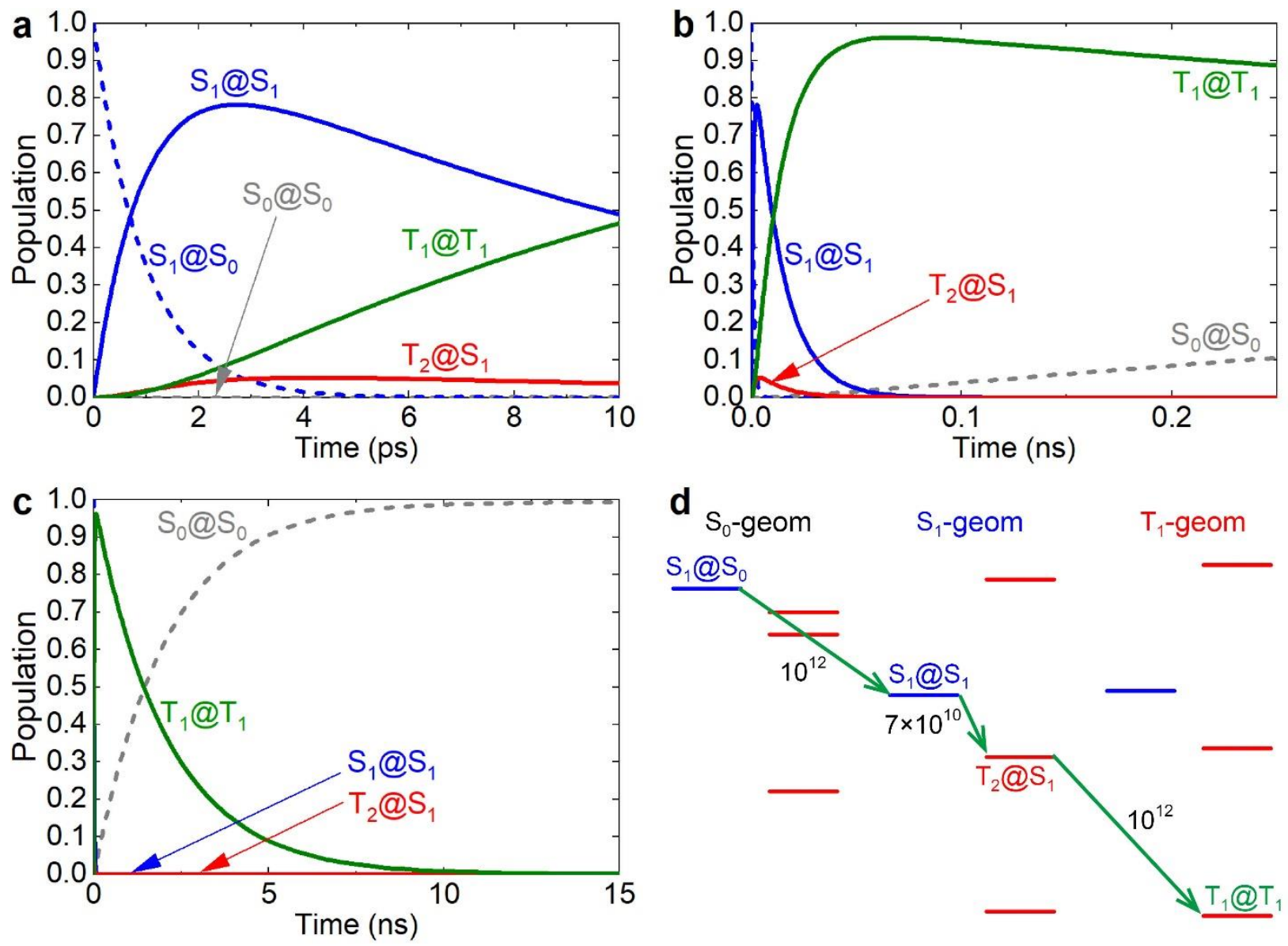

Figure S11. Calculated populations with the rate constants obtained with the Fourier transformed formulae when fwhm $=1400 \mathrm{~cm}^{-1}$ and $k_{\mathrm{GR}}=1 \times 10^{12} \mathrm{~s}^{-1}$ in the time range of (a) 0 10 ps, (b) 0-0.25 ns, and (c) 0-15 ns. (d) The proposed $\mathrm{T}_{2} @ \mathrm{~S}_{1}$ mediated $\mathrm{S}_{1} @ \mathrm{~S}_{0} \rightarrow \mathrm{S}_{1} @ \mathrm{~S}_{1} \rightarrow$ $\mathrm{T}_{2} @ \mathrm{~S}_{1} \rightarrow \mathrm{T}_{1} @ \mathrm{~T}_{1} \rightarrow \mathrm{S}_{0} @ \mathrm{~S}_{0}$ decay mechanism. 
Table S16. Nonradiative and radiative transition rate constants $\left(\mathrm{s}^{-1}\right)$ calculated for the $\mathrm{S}_{0}, \mathrm{~S}_{1}, \mathrm{~T}_{1}$, and $\mathrm{T}_{3}$ geometries of $\mathrm{BP}$ at the EOM-CCSD/6-31G(d) level of theory. The rate constants were calculated using the simplified method. The fwhm of the Lorentzian broadening was set to be $1000 \mathrm{~cm}^{-1}$.

\begin{tabular}{ccccc}
\hline \hline & $\mathrm{S}_{0}$ geom & $\mathrm{S}_{1}$ geom & $\mathrm{T}_{1}$ geom & $\mathrm{T}_{3}$ geom \\
\hline Nonradiative transition (ISC) & & & & \\
$\mathrm{S}_{1} \rightarrow \mathrm{T}_{1}$ & $4.1 \times 10^{6}$ & $1.7 \times 10^{7}$ & $4.3 \times 10^{7}$ & $7.5 \times 10^{6}$ \\
$\mathrm{~S}_{1} \rightarrow \mathrm{T}_{2}$ & $3.0 \times 10^{9}$ & $3.2 \times 10^{9}$ & $1.1 \times 10^{9}$ & $3.4 \times 10^{9}$ \\
$\mathrm{~S}_{1} \rightarrow \mathrm{T}_{3}$ & $2.1 \times 10^{8}$ & $6.0 \times 10^{1}$ & $1.8 \times 10^{2}$ & $1.2 \times 10^{8}$ \\
$\mathrm{~T}_{1} \rightarrow \mathrm{S}_{0}$ & $3.3 \times 10^{8}$ & $4.5 \times 10^{8}$ & $4.6 \times 10^{8}$ & $1.6 \times 10^{8}$ \\
$\mathrm{~T}_{1} \rightarrow \mathrm{S}_{1}$ & $4.8 \times 10^{-3}$ & $1.3 \times 10^{-1}$ & $1.8 \times 10^{-1}$ & $2.0 \times 10^{-4}$ \\
$\mathrm{~T}_{2} \rightarrow \mathrm{S}_{0}$ & $1.4 \times 10^{5}$ & $1.4 \times 10^{8}$ & $1.5 \times 10^{8}$ & $1.2 \times 10^{5}$ \\
$\mathrm{~T}_{2} \rightarrow \mathrm{S}_{1}$ & $2.8 \times 10^{6}$ & $1.3 \times 10^{9}$ & $1.3 \times 10^{9}$ & $1.2 \times 10^{4}$ \\
$\mathrm{~T}_{3} \rightarrow \mathrm{S}_{0}$ & $1.7 \times 10^{8}$ & $5.8 \times 10^{5}$ & $8.3 \times 10^{5}$ & $2.7 \times 10^{8}$ \\
$\mathrm{~T}_{3} \rightarrow \mathrm{S}_{1}$ & $3.8 \times 10^{5}$ & $1.7 \times 10^{8}$ & $2.6 \times 10^{8}$ & $1.1 \times 10^{4}$ \\
\hline $\mathrm{Nonradiative} \mathrm{transition} \mathrm{(IC)}$ & $3.2 \times 10^{6}$ & $3.5 \times 10^{7}$ & $4.8 \times 10^{7}$ & $1.2 \times 10^{7}$ \\
$\mathrm{~S}_{1} \rightarrow \mathrm{S}_{0}$ & $2.2 \times 10^{4}$ & $1.1 \times 10^{6}$ & $7.3 \times 10^{4}$ & $5.5 \times 10^{6}$ \\
$\mathrm{~T}_{1} \rightarrow \mathrm{T}_{2}$ & $3.3 \times 10^{6}$ & $2.4 \times 10^{-6}$ & $3.0 \times 10^{-7}$ & $1.6 \times 10^{6}$ \\
$\mathrm{~T}_{1} \rightarrow \mathrm{T}_{3}$ & $1.7 \times 10^{10}$ & $5.5 \times 10^{13}$ & $2.0 \times 10^{13}$ & $7.2 \times 10^{11}$ \\
$\mathrm{~T}_{2} \rightarrow \mathrm{T}_{1}$ & $8.6 \times 10^{13}$ & $4.8 \times 10^{4}$ & $2.5 \times 10^{4}$ & $3.9 \times 10^{10}$ \\
$\mathrm{~T}_{2} \rightarrow \mathrm{T}_{3}$ & $4.9 \times 10^{12}$ & $8.6 \times 10^{8}$ & $1.0 \times 10^{9}$ & $5.6 \times 10^{12}$ \\
$\mathrm{~T}_{3} \rightarrow \mathrm{T}_{1}$ & $1.6 \times 10^{14}$ & $3.3 \times 10^{11}$ & $3.0 \times 10^{11}$ & $1.1 \times 10^{12}$ \\
$\mathrm{~T}_{3} \rightarrow \mathrm{T}_{2}$ & & & & \\
Radiative decay & & & & \\
$\mathrm{S}_{1} \rightarrow \mathrm{S}_{0}$ (fluorescence) & $5.3 \times 10^{5}$ & $7.3 \times 10^{5}$ & $6.9 \times 10^{5}$ & $7.7 \times 10^{5}$ \\
$\mathrm{~T}_{1} \rightarrow \mathrm{S}_{0}$ (phosphorescence) & $6.9 \times 10^{1}$ & $8.0 \times 10^{2}$ & $7.2 \times 10^{2}$ & $5.0 \times 10^{2}$ \\
\hline \hline
\end{tabular}


Table S17. Nonradiative and radiative transition rate constants $\left(\mathrm{s}^{-1}\right)$ calculated for the $\mathrm{S}_{0}, \mathrm{~S}_{1}, \mathrm{~T}_{1}$, and $\mathrm{T}_{3}$ geometries of $\mathrm{BP}$ in $\mathrm{CH}_{2} \mathrm{Cl}_{2}$ solution (the (static) dielectric constant $\varepsilon=8.930000$; the optical dielectric constant $\varepsilon_{\infty}=2.028346$ ). The rate constants were calculated using the simplified method. The fwhm of the Lorentzian broadening was set to be $1000 \mathrm{~cm}^{-1}$.

\begin{tabular}{|c|c|c|c|c|}
\hline & $\mathrm{S}_{0}$ geom & $\mathrm{S}_{1}$ geom & $\mathrm{T}_{1}$ geom & $\mathrm{T}_{3}$ geom \\
\hline \multicolumn{5}{|l|}{ Nonradiative transition (ISC) } \\
\hline $\mathrm{S}_{1} \rightarrow \mathrm{T}_{1}$ & $8.7 \times 10^{8}$ & $1.4 \times 10^{9}$ & $1.5 \times 10^{9}$ & $1.6 \times 10^{9}$ \\
\hline $\mathrm{S}_{1} \rightarrow \mathrm{T}_{2}$ & $1.6 \times 10^{10}$ & $6.1 \times 10^{10}$ & $6.5 \times 10^{10}$ & $2.1 \times 10^{10}$ \\
\hline $\mathrm{S}_{1} \rightarrow \mathrm{T}_{3}$ & $5.8 \times 10^{9}$ & $3.1 \times 10^{6}$ & $6.5 \times 10^{6}$ & $5.2 \times 10^{9}$ \\
\hline $\mathrm{T}_{1} \rightarrow \mathrm{S}_{0}$ & $1.2 \times 10^{8}$ & $1.7 \times 10^{8}$ & $1.6 \times 10^{8}$ & $8.3 \times 10^{7}$ \\
\hline $\mathrm{T}_{1} \rightarrow \mathrm{S}_{1}$ & $7.3 \times 10^{-3}$ & $5.5 \times 10^{-3}$ & $2.1 \times 10^{-3}$ & $1.4 \times 10^{-3}$ \\
\hline $\mathrm{T}_{2} \rightarrow \mathrm{S}_{0}$ & $4.4 \times 10^{7}$ & $5.7 \times 10^{7}$ & $6.3 \times 10^{7}$ & $7.3 \times 10^{7}$ \\
\hline $\mathrm{T}_{2} \rightarrow \mathrm{S}_{1}$ & $2.6 \times 10^{6}$ & $5.8 \times 10^{6}$ & $1.5 \times 10^{7}$ & $6.4 \times 10^{4}$ \\
\hline $\mathrm{T}_{3} \rightarrow \mathrm{S}_{0}$ & $1.5 \times 10^{5}$ & $3.6 \times 10^{5}$ & $3.6 \times 10^{5}$ & $1.1 \times 10^{5}$ \\
\hline $\mathrm{T}_{3} \rightarrow \mathrm{S}_{1}$ & $2.0 \times 10^{6}$ & $2.8 \times 10^{9}$ & $4.1 \times 10^{9}$ & $3.7 \times 10^{3}$ \\
\hline \multicolumn{5}{|l|}{ Nonradiative transition (IC) } \\
\hline $\mathrm{S}_{1} \rightarrow \mathrm{S}_{0}$ & $4.6 \times 10^{6}$ & $4.5 \times 10^{6}$ & $4.0 \times 10^{6}$ & $4.3 \times 10^{6}$ \\
\hline $\mathrm{T}_{1} \rightarrow \mathrm{T}_{2}$ & $4.5 \times 10^{3}$ & $1.4 \times 10^{3}$ & $1.8 \times 10^{2}$ & $2.1 \times 10^{4}$ \\
\hline $\mathrm{T}_{1} \rightarrow \mathrm{T}_{3}$ & $3.1 \times 10^{2}$ & $1.8 \times 10^{-5}$ & $8.5 \times 10^{-6}$ & $1.5 \times 10^{5}$ \\
\hline $\mathrm{T}_{2} \rightarrow \mathrm{T}_{1}$ & $8.4 \times 10^{10}$ & $3.4 \times 10^{10}$ & $3.0 \times 10^{10}$ & $7.4 \times 10^{10}$ \\
\hline $\mathrm{T}_{2} \rightarrow \mathrm{T}_{3}$ & $1.3 \times 10^{14}$ & $7.9 \times 10^{4}$ & $2.8 \times 10^{5}$ & $3.8 \times 10^{12}$ \\
\hline $\mathrm{T}_{3} \rightarrow \mathrm{T}_{1}$ & $1.3 \times 10^{10}$ & $3.9 \times 10^{9}$ & $3.6 \times 10^{9}$ & $1.2 \times 10^{11}$ \\
\hline $\mathrm{T}_{3} \rightarrow \mathrm{T}_{2}$ & $2.8 \times 10^{14}$ & $7.5 \times 10^{11}$ & $7.5 \times 10^{11}$ & $8.7 \times 10^{11}$ \\
\hline \multicolumn{5}{|l|}{ Radiative decay } \\
\hline $\mathrm{S}_{1} \rightarrow \mathrm{S}_{0}$ (fluorescence) & $6.8 \times 10^{6}$ & $4.6 \times 10^{6}$ & $6.3 \times 10^{6}$ & $5.7 \times 10^{6}$ \\
\hline $\mathrm{T}_{1} \rightarrow \mathrm{S}_{0}$ (phosphorescence) & $3.9 \times 10^{2}$ & $7.9 \times 10^{2}$ & $8.7 \times 10^{2}$ & $5.2 \times 10^{2}$ \\
\hline
\end{tabular}


Table S18. Nonradiative and radiative transition rate constants $\left(\mathrm{s}^{-1}\right)$ calculated for the $\mathrm{S}_{0}, \mathrm{~S}_{1}, \mathrm{~T}_{1}$, and $\mathrm{T}_{3}$ geometries of $\mathrm{BP}$ in $\mathrm{CH}_{3} \mathrm{OH}$ solution (the (static) dielectric constant $\varepsilon=32.613000$; the optical dielectric constant $\left.\varepsilon_{\infty}=1.765709\right)$. The rate constants were calculated using the simplified method. The fwhm of the Lorentzian broadening was set to be $\mathbf{1 0 0 0} \mathrm{cm}^{-1}$.

\begin{tabular}{|c|c|c|c|c|}
\hline & $\mathrm{S}_{0}$ geom & $\mathrm{S}_{1}$ geom & $\mathrm{T}_{1}$ geom & $\mathrm{T}_{3}$ geom \\
\hline \multicolumn{5}{|l|}{ Nonradiative transition (ISC) } \\
\hline $\mathrm{S}_{1} \rightarrow \mathrm{T}_{1}$ & $9.3 \times 10^{8}$ & $1.5 \times 10^{9}$ & $1.5 \times 10^{9}$ & $1.7 \times 10^{9}$ \\
\hline $\mathrm{S}_{1} \rightarrow \mathrm{T}_{2}$ & $1.5 \times 10^{10}$ & $6.0 \times 10^{10}$ & $6.0 \times 10^{10}$ & $2.1 \times 10^{10}$ \\
\hline $\mathrm{S}_{1} \rightarrow \mathrm{T}_{3}$ & $4.1 \times 10^{9}$ & $6.7 \times 10^{6}$ & $1.7 \times 10^{7}$ & $3.9 \times 10^{9}$ \\
\hline $\mathrm{T}_{1} \rightarrow \mathrm{S}_{0}$ & $1.1 \times 10^{8}$ & $1.6 \times 10^{8}$ & $1.5 \times 10^{8}$ & $7.7 \times 10^{7}$ \\
\hline $\mathrm{T}_{1} \rightarrow \mathrm{S}_{1}$ & $7.0 \times 10^{-3}$ & $6.1 \times 10^{-3}$ & $2.3 \times 10^{-3}$ & $1.2 \times 10^{-3}$ \\
\hline $\mathrm{T}_{2} \rightarrow \mathrm{S}_{0}$ & $4.6 \times 10^{7}$ & $5.5 \times 10^{7}$ & $6.5 \times 10^{7}$ & $7.7 \times 10^{7}$ \\
\hline $\mathrm{T}_{2} \rightarrow \mathrm{S}_{1}$ & $1.7 \times 10^{6}$ & $4.8 \times 10^{6}$ & $1.2 \times 10^{7}$ & $5.2 \times 10^{4}$ \\
\hline $\mathrm{T}_{3} \rightarrow \mathrm{S}_{0}$ & $1.5 \times 10^{5}$ & $3.3 \times 10^{5}$ & $3.3 \times 10^{5}$ & $9.1 \times 10^{4}$ \\
\hline $\mathrm{T}_{3} \rightarrow \mathrm{S}_{1}$ & $7.1 \times 10^{5}$ & $2.1 \times 10^{9}$ & $2.8 \times 10^{9}$ & $5.2 \times 10^{4}$ \\
\hline \multicolumn{5}{|l|}{ Nonradiative transition (IC) } \\
\hline $\mathrm{S}_{1} \rightarrow \mathrm{S}_{0}$ & $4.6 \times 10^{6}$ & $3.7 \times 10^{6}$ & $4.0 \times 10^{6}$ & $3.9 \times 10^{6}$ \\
\hline $\mathrm{T}_{1} \rightarrow \mathrm{T}_{2}$ & $6.5 \times 10^{3}$ & $1.7 \times 10^{3}$ & $2.4 \times 10^{2}$ & $2.1 \times 10^{4}$ \\
\hline $\mathrm{T}_{1} \rightarrow \mathrm{T}_{3}$ & $7.0 \times 10^{2}$ & $6.6 \times 10^{-5}$ & $4.1 \times 10^{-5}$ & $2.0 \times 10^{5}$ \\
\hline $\mathrm{T}_{2} \rightarrow \mathrm{T}_{1}$ & $9.4 \times 10^{10}$ & $3.3 \times 10^{10}$ & $3.2 \times 10^{10}$ & $7.6 \times 10^{10}$ \\
\hline $\mathrm{T}_{2} \rightarrow \mathrm{T}_{3}$ & $5.0 \times 10^{14}$ & $2.1 \times 10^{5}$ & $1.2 \times 10^{6}$ & $2.4 \times 10^{12}$ \\
\hline $\mathrm{T}_{3} \rightarrow \mathrm{T}_{1}$ & $1.6 \times 10^{10}$ & $5.0 \times 10^{9}$ & $4.7 \times 10^{9}$ & $9.8 \times 10^{10}$ \\
\hline $\mathrm{T}_{3} \rightarrow \mathrm{T}_{2}$ & $8.0 \times 10^{14}$ & $8.2 \times 10^{11}$ & $9.9 \times 10^{11}$ & $3.2 \times 10^{11}$ \\
\hline \multicolumn{5}{|l|}{ Radiative decay } \\
\hline $\mathrm{S}_{1} \rightarrow \mathrm{S}_{0}$ (fluorescence) & $6.5 \times 10^{6}$ & $4.3 \times 10^{6}$ & $5.9 \times 10^{6}$ & $5.4 \times 10^{6}$ \\
\hline $\mathrm{T}_{1} \rightarrow \mathrm{S}_{0}$ (phosphorescence) & $4.0 \times 10^{2}$ & $7.7 \times 10^{2}$ & $9.0 \times 10^{2}$ & $5.3 \times 10^{2}$ \\
\hline
\end{tabular}


Table S19. Nonradiative and radiative transition rate constants $\left(\mathrm{s}^{-1}\right)$ calculated for the $\mathrm{S}_{0}, \mathrm{~S}_{1}, \mathrm{~T}_{1}$, and $\mathrm{T}_{3}$ geometries of $\mathrm{BP}$ in $\mathrm{CH}_{3} \mathrm{CN}$ solution (the (static) dielectric constant $\varepsilon=35.688000$; the optical dielectric constant $\left.\varepsilon_{\infty}=1.806874\right)$. The rate constants were calculated using the simplified method. The fwhm of the Lorentzian broadening was set to be $\mathbf{1 0 0 0} \mathrm{cm}^{-1}$.

\begin{tabular}{|c|c|c|c|c|}
\hline & $\mathrm{S}_{0}$ geom & $\mathrm{S}_{1}$ geom & $\mathrm{T}_{1}$ geom & $\mathrm{T}_{3}$ geom \\
\hline \multicolumn{5}{|l|}{ Nonradiative transition (ISC) } \\
\hline $\mathrm{S}_{1} \rightarrow \mathrm{T}_{1}$ & $9.3 \times 10^{8}$ & $1.5 \times 10^{9}$ & $1.5 \times 10^{9}$ & $1.7 \times 10^{9}$ \\
\hline $\mathrm{S}_{1} \rightarrow \mathrm{T}_{2}$ & $1.5 \times 10^{10}$ & $6.0 \times 10^{10}$ & $6.0 \times 10^{10}$ & $2.1 \times 10^{10}$ \\
\hline $\mathrm{S}_{1} \rightarrow \mathrm{T}_{3}$ & $4.0 \times 10^{9}$ & $6.8 \times 10^{6}$ & $1.7 \times 10^{7}$ & $3.9 \times 10^{9}$ \\
\hline $\mathrm{T}_{1} \rightarrow \mathrm{S}_{0}$ & $1.1 \times 10^{8}$ & $1.6 \times 10^{8}$ & $1.5 \times 10^{8}$ & $7.7 \times 10^{7}$ \\
\hline $\mathrm{T}_{1} \rightarrow \mathrm{S}_{1}$ & $7.0 \times 10^{-3}$ & $6.2 \times 10^{-3}$ & $2.3 \times 10^{-3}$ & $1.2 \times 10^{-3}$ \\
\hline $\mathrm{T}_{2} \rightarrow \mathrm{S}_{0}$ & $4.6 \times 10^{7}$ & $5.5 \times 10^{7}$ & $6.5 \times 10^{7}$ & $7.7 \times 10^{7}$ \\
\hline $\mathrm{T}_{2} \rightarrow \mathrm{S}_{1}$ & $1.7 \times 10^{6}$ & $4.9 \times 10^{6}$ & $1.2 \times 10^{7}$ & $5.2 \times 10^{4}$ \\
\hline $\mathrm{T}_{3} \rightarrow \mathrm{S}_{0}$ & $1.5 \times 10^{5}$ & $3.3 \times 10^{5}$ & $3.3 \times 10^{5}$ & $8.7 \times 10^{5}$ \\
\hline $\mathrm{T}_{3} \rightarrow \mathrm{S}_{1}$ & $6.9 \times 10^{5}$ & $2.1 \times 10^{9}$ & $2.8 \times 10^{9}$ & $1.3 \times 10^{3}$ \\
\hline \multicolumn{5}{|l|}{ Nonradiative transition (IC) } \\
\hline $\mathrm{S}_{1} \rightarrow \mathrm{S}_{0}$ & $4.6 \times 10^{6}$ & $3.7 \times 10^{6}$ & $4.0 \times 10^{6}$ & $3.9 \times 10^{6}$ \\
\hline $\mathrm{T}_{1} \rightarrow \mathrm{T}_{2}$ & $6.5 \times 10^{3}$ & $1.7 \times 10^{3}$ & $2.4 \times 10^{2}$ & $2.1 \times 10^{4}$ \\
\hline $\mathrm{T}_{1} \rightarrow \mathrm{T}_{3}$ & $7.2 \times 10^{2}$ & $6.9 \times 10^{-5}$ & $4.4 \times 10^{-5}$ & $2.1 \times 10^{5}$ \\
\hline $\mathrm{T}_{2} \rightarrow \mathrm{T}_{1}$ & $9.4 \times 10^{10}$ & $3.3 \times 10^{10}$ & $3.3 \times 10^{10}$ & $7.6 \times 10^{10}$ \\
\hline $\mathrm{T}_{2} \rightarrow \mathrm{T}_{3}$ & $5.4 \times 10^{14}$ & $2.1 \times 10^{5}$ & $1.3 \times 10^{6}$ & $2.4 \times 10^{12}$ \\
\hline $\mathrm{T}_{3} \rightarrow \mathrm{T}_{1}$ & $1.6 \times 10^{10}$ & $5.1 \times 10^{9}$ & $4.8 \times 10^{9}$ & $9.8 \times 10^{10}$ \\
\hline $\mathrm{T}_{3} \rightarrow \mathrm{T}_{2}$ & $8.5 \times 10^{14}$ & $8.2 \times 10^{11}$ & $1.0 \times 10^{12}$ & $3.2 \times 10^{11}$ \\
\hline \multicolumn{5}{|l|}{ Radiative decay } \\
\hline $\mathrm{S}_{1} \rightarrow \mathrm{S}_{0}$ (fluorescence) & $6.5 \times 10^{6}$ & $4.3 \times 10^{6}$ & $6.0 \times 10^{6}$ & $5.5 \times 10^{6}$ \\
\hline $\mathrm{T}_{1} \rightarrow \mathrm{S}_{0}$ (phosphorescence) & $4.1 \times 10^{2}$ & $7.7 \times 10^{2}$ & $9.0 \times 10^{2}$ & $5.4 \times 10^{2}$ \\
\hline
\end{tabular}


Table S20. Nonradiative and radiative transition rate constants $\left(\mathrm{s}^{-1}\right)$ calculated for the $\mathrm{S}_{0}, \mathrm{~S}_{1}, \mathrm{~T}_{1}$, and $\mathrm{T}_{3}$ geometries of $\mathrm{BP}$ in mixture of $\mathrm{C}_{2} \mathrm{H}_{5} \mathrm{OC}_{2} \mathrm{H}_{5}, n$-pentane, and $\mathrm{C}_{2} \mathrm{H}_{5} \mathrm{OH}$ solution (the (static) dielectric constant $\varepsilon=6.674152$; the optical dielectric constant $\varepsilon_{\infty}=1.838904$ ). The mixed solution mimics EPA solution used in ref 25 of the main text. The rate constants were calculated

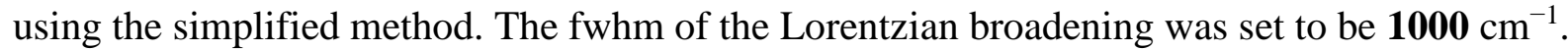
The temperature was set to be $\mathbf{3 0 0} \mathrm{K}$.

\begin{tabular}{|c|c|c|c|c|}
\hline & $\mathrm{S}_{0}$ geom & $\mathrm{S}_{1}$ geom & $\mathrm{T}_{1}$ geom & $\mathrm{T}_{3}$ geom \\
\hline \multicolumn{5}{|l|}{$\begin{array}{l}\text { Nonradiative transition (ISC) } \\
\text { (ISC) }\end{array}$} \\
\hline $\mathrm{S}_{1} \rightarrow \mathrm{T}_{1}$ & $8.5 \times 10^{8}$ & $1.4 \times 10^{9}$ & $1.4 \times 10^{9}$ & $1.6 \times 10^{9}$ \\
\hline $\mathrm{S}_{1} \rightarrow \mathrm{T}_{2}$ & $1.7 \times 10^{10}$ & $6.3 \times 10^{10}$ & $6.6 \times 10^{10}$ & $2.1 \times 10^{10}$ \\
\hline $\mathrm{S}_{1} \rightarrow \mathrm{T}_{3}$ & $6.8 \times 10^{9}$ & $2.4 \times 10^{6}$ & $4.5 \times 10^{6}$ & $6.1 \times 10^{9}$ \\
\hline $\mathrm{T}_{1} \rightarrow \mathrm{S}_{0}$ & $1.2 \times 10^{8}$ & $1.7 \times 10^{8}$ & $1.7 \times 10^{8}$ & $8.5 \times 10^{7}$ \\
\hline $\mathrm{T}_{1} \rightarrow \mathrm{S}_{1}$ & $7.2 \times 10^{-3}$ & $5.3 \times 10^{-3}$ & $2.0 \times 10^{-3}$ & $1.5 \times 10^{-3}$ \\
\hline $\mathrm{T}_{2} \rightarrow \mathrm{S}_{0}$ & $4.4 \times 10^{7}$ & $5.7 \times 10^{7}$ & $6.2 \times 10^{7}$ & $7.3 \times 10^{7}$ \\
\hline $\mathrm{T}_{2} \rightarrow \mathrm{S}_{1}$ & $3.0 \times 10^{6}$ & $6.3 \times 10^{6}$ & $1.6 \times 10^{7}$ & $6.9 \times 10^{4}$ \\
\hline $\mathrm{T}_{3} \rightarrow \mathrm{S}_{0}$ & $1.5 \times 10^{5}$ & $3.7 \times 10^{5}$ & $3.8 \times 10^{5}$ & $1.1 \times 10^{5}$ \\
\hline $\mathrm{T}_{3} \rightarrow \mathrm{S}_{1}$ & $3.1 \times 10^{6}$ & $3.3 \times 10^{9}$ & $4.7 \times 10^{9}$ & $5.7 \times 10^{3}$ \\
\hline \multicolumn{5}{|l|}{ Nonradiative transition (IC) } \\
\hline $\mathrm{S}_{1} \rightarrow \mathrm{S}_{0}$ & $4.6 \times 10^{6}$ & $4.4 \times 10^{6}$ & $4.0 \times 10^{6}$ & $4.3 \times 10^{6}$ \\
\hline $\mathrm{T}_{1} \rightarrow \mathrm{T}_{2}$ & $3.8 \times 10^{3}$ & $1.3 \times 10^{3}$ & $1.6 \times 10^{2}$ & $2.1 \times 10^{4}$ \\
\hline $\mathrm{T}_{1} \rightarrow \mathrm{T}_{3}$ & $2.1 \times 10^{2}$ & $1.0 \times 10^{-5}$ & $4.3 \times 10^{-6}$ & $1.6 \times 10^{5}$ \\
\hline $\mathrm{T}_{2} \rightarrow \mathrm{T}_{1}$ & $8.1 \times 10^{10}$ & $3.3 \times 10^{10}$ & $2.8 \times 10^{10}$ & $7.5 \times 10^{10}$ \\
\hline $\mathrm{T}_{2} \rightarrow \mathrm{T}_{3}$ & $7.9 \times 10^{13}$ & $5.0 \times 10^{4}$ & $1.5 \times 10^{5}$ & $4.9 \times 10^{12}$ \\
\hline $\mathrm{T}_{3} \rightarrow \mathrm{T}_{1}$ & $1.2 \times 10^{10}$ & $3.6 \times 10^{9}$ & $3.3 \times 10^{9}$ & $1.6 \times 10^{11}$ \\
\hline $\mathrm{T}_{3} \rightarrow \mathrm{T}_{2}$ & $2.0 \times 10^{14}$ & $6.9 \times 10^{11}$ & $6.6 \times 10^{11}$ & $1.4 \times 10^{12}$ \\
\hline \multicolumn{5}{|l|}{ Radiative decay } \\
\hline $\mathrm{S}_{1} \rightarrow \mathrm{S}_{0}$ (fluorescence) & $6.5 \times 10^{6}$ & $4.4 \times 10^{6}$ & $6.0 \times 10^{6}$ & $5.4 \times 10^{6}$ \\
\hline $\mathrm{T}_{1} \rightarrow \mathrm{S}_{0}$ (phosphorescence) & $3.8 \times 10^{2}$ & $7.8 \times 10^{2}$ & $8.5 \times 10^{2}$ & $5.2 \times 10^{2}$ \\
\hline
\end{tabular}


Table S21. Nonradiative and radiative transition rate constants $\left(\mathrm{s}^{-1}\right)$ calculated for the $\mathrm{S}_{0}, \mathrm{~S}_{1}, \mathrm{~T}_{1}$, and $\mathrm{T}_{3}$ geometries of $\mathrm{BP}$ in mixture of $\mathrm{C}_{2} \mathrm{H}_{5} \mathrm{OC}_{2} \mathrm{H}_{5}, n$-pentane, and $\mathrm{C}_{2} \mathrm{H}_{5} \mathrm{OH}$ solution (the (static) dielectric constant $\varepsilon=6.674152$; the optical dielectric constant $\varepsilon_{\infty}=1.838904$ ). The mixed solution mimics EPA solution used in ref 25 of the main text. The rate constants were calculated

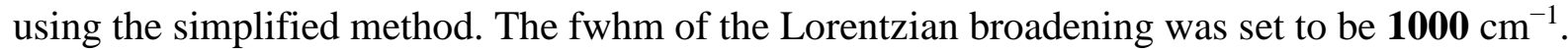
The temperature was set to be $\mathbf{7 7} \mathbf{K}$.

\begin{tabular}{|c|c|c|c|c|}
\hline & $\mathrm{S}_{0}$ geom & $\mathrm{S}_{1}$ geom & $\mathrm{T}_{1}$ geom & $\mathrm{T}_{3}$ geom \\
\hline \multicolumn{5}{|l|}{ Nonradiative transition (ISC) } \\
\hline $\mathrm{S}_{1} \rightarrow \mathrm{T}_{1}$ & $8.5 \times 10^{8}$ & $1.4 \times 10^{9}$ & $1.4 \times 10^{9}$ & $1.6 \times 10^{9}$ \\
\hline $\mathrm{S}_{1} \rightarrow \mathrm{T}_{2}$ & $1.7 \times 10^{10}$ & $6.3 \times 10^{10}$ & $6.6 \times 10^{10}$ & $2.1 \times 10^{10}$ \\
\hline $\mathrm{S}_{1} \rightarrow \mathrm{T}_{3}$ & $6.8 \times 10^{9}$ & $8.4 \times 10^{-5}$ & $3.1 \times 10^{-4}$ & $6.1 \times 10^{9}$ \\
\hline $\mathrm{T}_{1} \rightarrow \mathrm{S}_{0}$ & $1.2 \times 10^{8}$ & $1.7 \times 10^{8}$ & $1.7 \times 10^{8}$ & $8.5 \times 10^{7}$ \\
\hline $\mathrm{T}_{1} \rightarrow \mathrm{S}_{1}$ & 0.0 & 0.0 & 0.0 & 0.0 \\
\hline $\mathrm{T}_{2} \rightarrow \mathrm{S}_{0}$ & $4.4 \times 10^{7}$ & $5.7 \times 10^{7}$ & $6.2 \times 10^{7}$ & $7.3 \times 10^{7}$ \\
\hline $\mathrm{T}_{2} \rightarrow \mathrm{S}_{1}$ & $1.1 \times 10^{-3}$ & $3.9 \times 10^{-4}$ & $1.4 \times 10^{-2}$ & 0.0 \\
\hline $\mathrm{T}_{3} \rightarrow \mathrm{S}_{0}$ & $1.5 \times 10^{5}$ & $3.7 \times 10^{5}$ & $3.8 \times 10^{5}$ & $1.1 \times 10^{5}$ \\
\hline $\mathrm{T}_{3} \rightarrow \mathrm{S}_{1}$ & $1.6 \times 10^{-2}$ & $3.3 \times 10^{9}$ & $4.7 \times 10^{9}$ & 0.0 \\
\hline \multicolumn{5}{|l|}{ Nonradiative transition (IC) } \\
\hline $\mathrm{S}_{1} \rightarrow \mathrm{S}_{0}$ & $3.0 \times 10^{6}$ & $3.1 \times 10^{6}$ & $2.8 \times 10^{6}$ & $3.0 \times 10^{6}$ \\
\hline $\mathrm{T}_{1} \rightarrow \mathrm{T}_{2}$ & 0.0 & 0.0 & 0.0 & 0.0 \\
\hline $\mathrm{T}_{1} \rightarrow \mathrm{T}_{3}$ & 0.0 & 0.0 & 0.0 & 0.0 \\
\hline $\mathrm{T}_{2} \rightarrow \mathrm{T}_{1}$ & $6.6 \times 10^{10}$ & $2.9 \times 10^{10}$ & $2.5 \times 10^{10}$ & $6.3 \times 10^{10}$ \\
\hline $\mathrm{T}_{2} \rightarrow \mathrm{T}_{3}$ & $5.4 \times 10^{12}$ & 0.0 & 0.0 & $3.5 \times 10^{12}$ \\
\hline $\mathrm{T}_{3} \rightarrow \mathrm{T}_{1}$ & $1.1 \times 10^{10}$ & $3.4 \times 10^{9}$ & $3.1 \times 10^{9}$ & $1.6 \times 10^{11}$ \\
\hline $\mathrm{T}_{3} \rightarrow \mathrm{T}_{2}$ & $2.0 \times 10^{14}$ & $6.5 \times 10^{11}$ & $6.4 \times 10^{11}$ & $2.7 \times 10^{10}$ \\
\hline \multicolumn{5}{|l|}{ Radiative decay } \\
\hline $\mathrm{S}_{1} \rightarrow \mathrm{S}_{0}$ (fluorescence) & $6.5 \times 10^{6}$ & $4.4 \times 10^{6}$ & $6.0 \times 10^{6}$ & $5.4 \times 10^{6}$ \\
\hline $\mathrm{T}_{1} \rightarrow \mathrm{S}_{0}$ (phosphorescence) & $3.8 \times 10^{2}$ & $7.8 \times 10^{2}$ & $8.5 \times 10^{2}$ & $5.2 \times 10^{2}$ \\
\hline
\end{tabular}

